\title{
The vortex liquid piston engine and some other vortex technologies
}

\author{
M GOLDSHTIK, F HUSSAIN and R J YAO \\ Department of Mechanical Engineering, University of Houston, Houston, \\ TX 77204-4792, USA \\ e-mail: FHussain@uh.edu
}

\begin{abstract}
By exploiting three unique characteristics of confined swirling incompressible flows - centrifugal acceleration, internal separation or recirculation zones near the axis, and bistability (i.e. rarefied and condensed stable states) of multi-phase flows - we developed several innovative vortex machines which will revolutionize mechanical technologies in a variety of industries. The machines utilizing these features include: Vortex Engine, Vortex Thruster, Vortex Suction Device, Vortex Chemical Reactor, Bubbling Centrifuge and Vortex Mill. As a specific example, we describe here in some detail the development of a liquid piston engine, including analysis of its hydrodynamic and thermodynamic features. We have designed a laboratory 'cold' model and performed detailed experimental, theoretical and numerical analyses to study the role of the controlling parameters and are now ready to test a 'hot' model. In addition, we mention a few other vortex technologies of interest to us.
\end{abstract}

Keywords. Multi-phase flows; incompressible flows; vortex machines; vortex liquid piston engine; swirling flows.

\section{Introduction: Rotating single- and multi-phase flows}

While the proposed applications are innovative, rotating flows within vortex chambers have been investigated extensively. As shown schematically in figure 1, the tangential entry of fluid into a typical cylindrical vortex chamber, via the guiding vanes around the periphery, causes swirling motion of the fluid (mixture). In the core region of the flow away from the walls, the radial pressure gradient balances the centrifugal force; this is the so-called cyclostrophic balance. Near the end walls, the centrifugal acceleration is very small due to the boundary layer effect but the radial pressure gradient is essentially the same as in the core region. Thus, the lack of cyclostrophic balance causes the pressure gradient to

A list of symbols used appears at the end of the paper. 


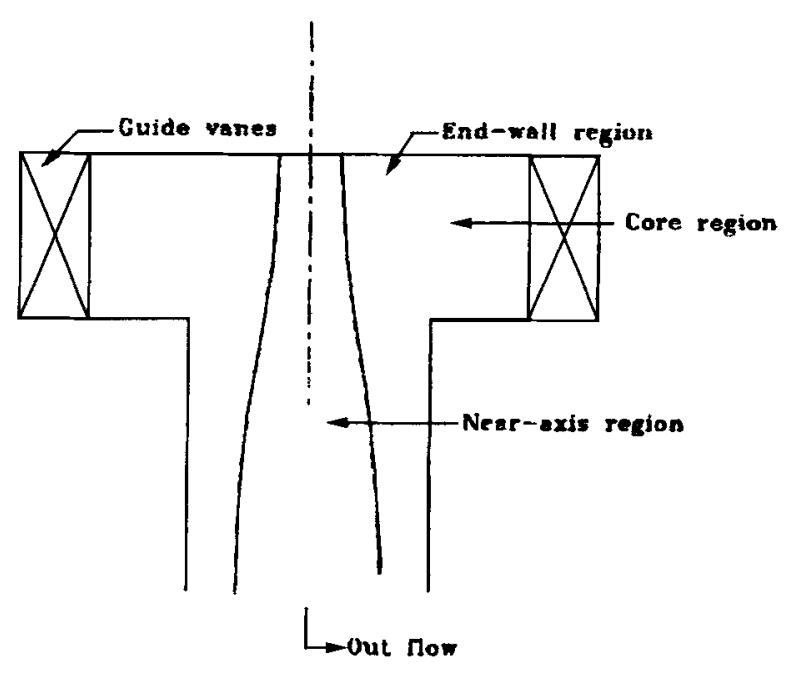

Figure 1. Schematic of various flow regions in an axisymmetric vortex chamber.

generate a secondary flow towards the axis along the end walls, balanced by the frictional force. This leads to an end-wall boundary layer with a substantial inflow radial speed $v_{r}$, the maximum of $v_{r}$ (and of axial and azimuthal velocities) occurring inside the boundary layer. This inflow of the rotating fluid along the end wall prevents the typically desirable prolonged retention of small particles within the chamber, because they are carried away by the fluid flow along the axis. However, by appropriately profiling the end walls (we have developed an equation for the end wall profile using hydrodynamic analysis and laboratory tests), small particles can be retained within the chamber for longer durations. At practical operating speeds, the flow in the near-axis region is extremely complex, involving very high turbulence and noise-producing axial oscillations. A detailed study of the flow patterns in

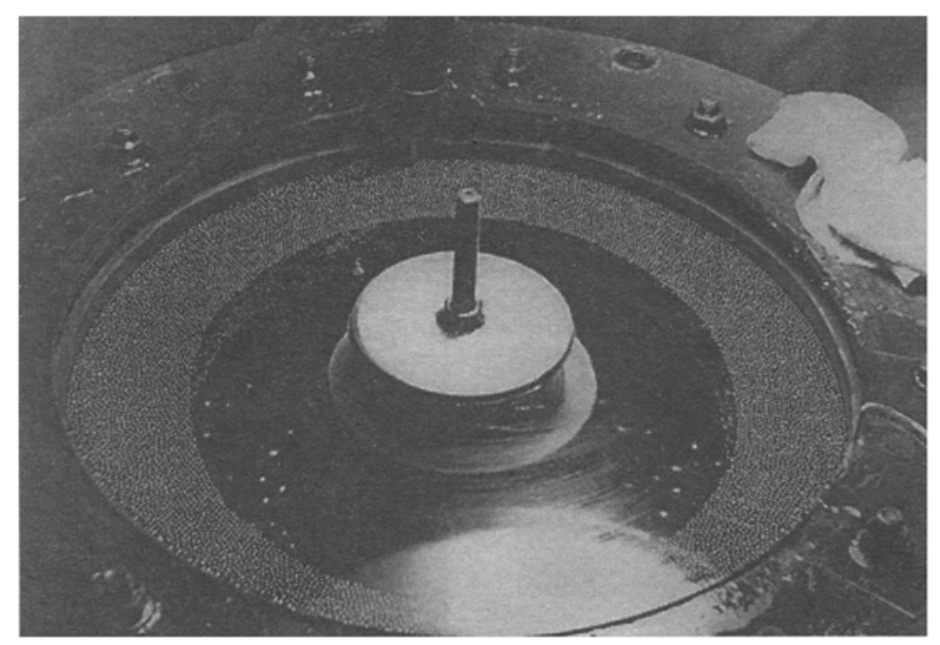

Figure 2. Condensed stable state of a mixture of liquid-solid particle in a vortex chamber. Note the dense, homogeneous particle layer near the periphery, forming a rotating axisymmetric fluidized bed. 


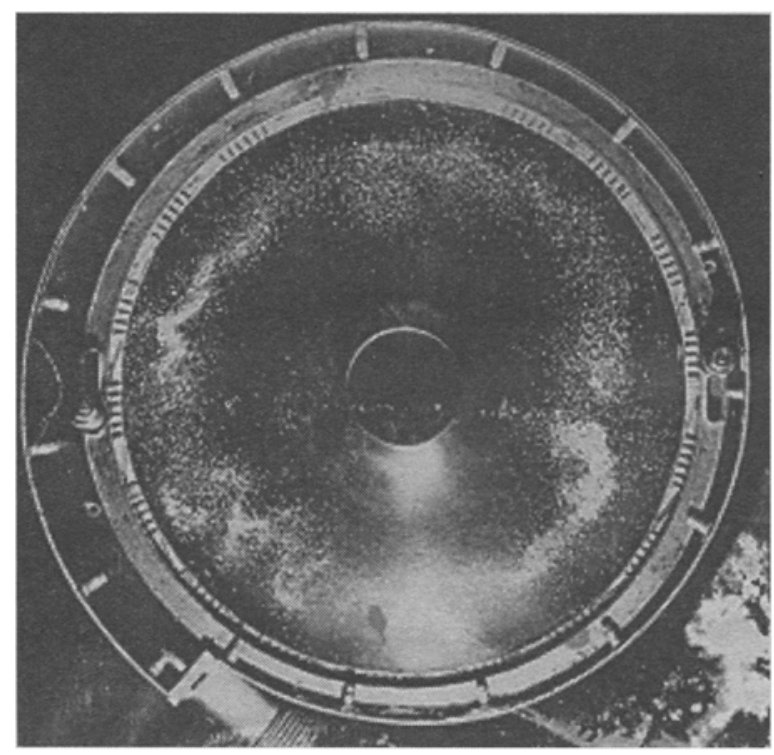

Figure 3. Rarefied stable state with small particles rotating in circular trajectories in a vortex chamber.

this and other regions of the vortex chamber for various operating parameters is necessary to develop more efficient designs.

Experimental and theoretical studies (Batchelor 1967; Greenspan 1969; Goldshtik 1981, 1984; Gupta et al 1984; Hussain 1986) show that rotating flows possess three fundamental features, described below.

High centrifugal force: Typical inlet gas velocities $(\approx 100 \mathrm{~m} / \mathrm{s})$ into a chamber of radius $\approx 0.1 \mathrm{~m}$ produce a centrifugal acceleration $a_{c} \approx 10^{4} \mathrm{~g}$. This high centrifugal force is central to all applications discussed here, and ensures stability of the central cavity and motion of solid particles/fluid bubbles in circular trajectories inside the cylindrical vortex chamber.

Near-axis flow: Near the axis of rotation, if the speed is high enough, a gaseous cavity is formed when a liquid is used as the working fluid, and a recirculation zone (such as vortex breakdown bubble or internal separation, i.e. separation away from any wall), forms if the fluid is a gas. The complex flow pattern in this region depends on the operating conditions (such as Reynolds number and Rossby number) and the chamber end-wall profile; and considerably influences the flow in the rest of the chamber. A clear understanding of the flow in this region based on rigorous hydrodynamic analysis is essential for optimal design of these machines, particularly the vortex engine and vortex thruster.

Bistability: When two-phase flow is involved, our experiments in vortex chambers have shown the existence of two stable states, either condensed or rarefied. 


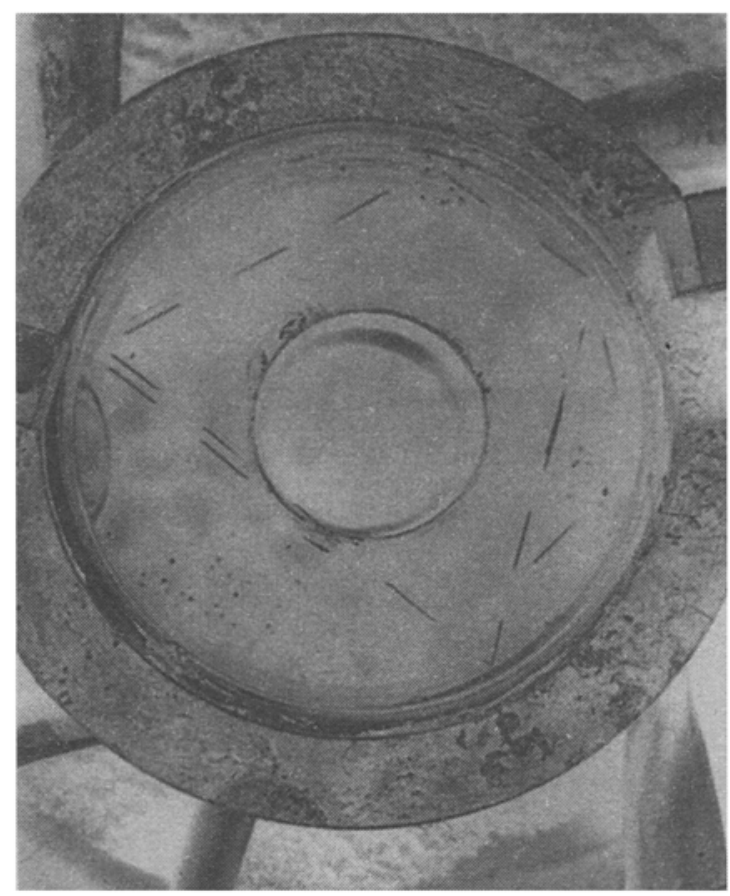

Figure 4. Rarefied stable state with large particles, which move in polygonal paths and collide with the outer wall, forming the basis for a vortex mill.

Condensed stable state: At high particle concentrations, the denser medium (solid or liquid) forms a tightly packed layer near the periphery of the vortex chamber and provides a very large interface area between the two media; figure 2 illustrates a condensed particle layer.

Rarefied stable state: At lower concentrations, the particles are distributed homogeneously and move in circular orbits in the lighter fluid medium (figures 3,4 ). The radii of the orbits depend on the particle size. For solids, larger particles collide with the chamber walls and are ground to smaller sizes (figure 4); this leads to the proposed vortex mill (see $\S 8$ ).

In this paper we focus entirely on the liquid piston engine; the other machines will be considered very briefly at the end.

\section{Vortex engine}

Conventional piston engines have relatively complicated designs and undergo greater wear and tear due to moving mechanical parts. An external combustion, flat-top liquid piston Stirling engine (West 1983) is very simple and does not employ complicated mechanical parts. The main drawback of such an engine is the instability of the liquid piston's top surface. When the piston is near its top dead centre, its speed becomes zero, but acceleration is maximum. Under these conditions, a stability criterion is that the ratio of gravitational 


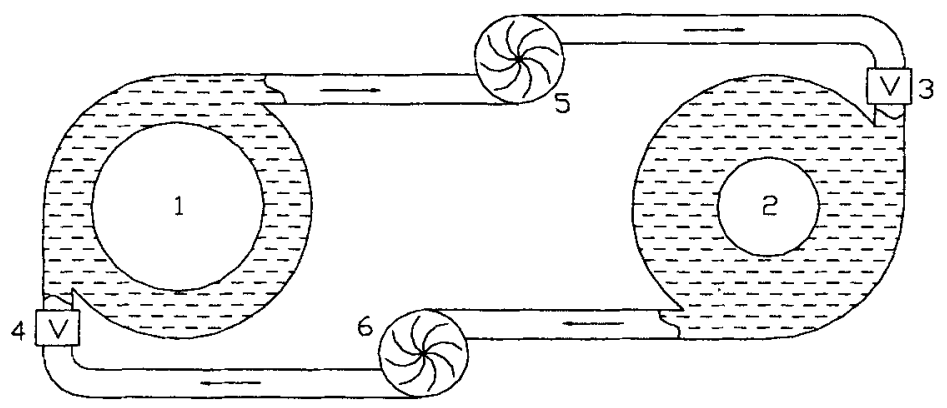

Figure 5. Working principle of the liquid piston engine (see Goldshtik 1992); 1, 2-oscillating cavities; 3,4 - check valves; 5,6 - hydromotors.

acceleration to fluid acceleration must be greater than unity so that no liquid will leave the surface of the liquid piston. Therefore, the frequency of the engine must be less than one hertz in order to avoid the instability of the flat liquid piston surface. Accordingly, the efficiency of this engine is very low, about one percent, and the power of this engine is only several watts at best.

These limitations of the flat top liquid piston engine are overcome by employing a rotating liquid piston (Goldshtik 1992), where centrifugal acceleration is used instead of gravity. This is an internal combustion engine with two circular cylinders (i.e. vortex chambers) that are partially filled with a fixed volume of liquid and are connected tangentially by two channels containing hydromotors (see figure 5). Each of these circular cylinders has a top and a bottom cover, a system for intake of fuel and air, and an associated exhaust system. Each cylinder may have either an electric spark plug or may work in diesel mode (via a fuel spray injector). By using tangential entry, the liquid rotates at high speed within the cylinder and creates a vertical cylindrical gas cavity around the axis of the rotating liquid. Rotation is used to stabilize the liquid-gas interface of the cylindrical cavity, which functions like the piston top in a conventional internal combustion engine compressing the fuel-air mixture. This cavity is the combustion chamber into which the fuel-air mixture is injected. When the mixture is ignited, the increased pressure in the cavity forces some of the liquid out through a tangential channel, through a hydromotor, and then tangentially into the second cylinder wherein it keeps its swirling motion; this sequence is repeated in the second cylinder and the liquid then flows back into the first cylinder. In this manner, as a result of the pressure from combustion, the liquid is transferred back and forth between the two cylinders at a frequency that can be controlled by changing the system parameters. The unidirectionally rotating hydromotors extract energy for mechanical drive. In summary, this device works without any moving mechanical part, with the exception of the hydromotors and valves to control flow timing of liquid, fuel/air mixture, and products of combustion.

In this paper, we focus on the detailed theoretical description of the engine, and also present some preliminary numerical and experimental results. Results of the engine's numerical optimization and experimental investigations will be published in the future. 


\section{Simple theoretical model of vortex liquid piston engine (VLPE)}

\subsection{Principal dynamical equation}

The model assumes an unsteady flow of a liquid in two identical interconnected cylinders, with near-axis cylindrical cavities filled with an ideal gas and having time-dependent radii $r_{1}(t)$ and $r_{2}(t)$. The flow between the cylinders generates a self-sustained oscillation due to combustion-induced thermal expansion and contraction processes running in opposite phases. As a first step, viscosity is neglected; viscous effects are considered later in both theory and numerical simulations.

For axisymmetric flow, we have the following relationships for radial and tangential velocities:

$$
v_{r}=Q / r \text { and } v_{\varphi}=\Gamma / r
$$

where $Q=Q(t)$ and $\Gamma=$ const. are related to the dimensional physical volume flow rate $Q_{v}$ and circulation $\Gamma_{R}$ by

$$
Q_{v}=2 \pi h Q \quad \text { and } \quad \Gamma_{R}=2 \pi \Gamma,
$$

where $h$ is the chamber height. Conservation of the fluid volume gives the relation

$$
r_{1}^{2}+r_{2}^{2}=2 \sigma R^{2}
$$

where $R$ is the cylinder radius and $\sigma$ is the total gas/cylinder volume ratio $(1-\sigma$ is the total volume fraction of liquid). The radial velocity of the liquid-gas surface is $v_{r}=\mathrm{d} r / \mathrm{d} t$. From (1), we have

$$
Q=r(\mathrm{~d} r / \mathrm{d} t)
$$

With the help of (3) and (4) we can obtain

$$
Q_{1}=-Q_{2}=Q
$$

For an incompressible, inviscid liquid in unsteady, axisymmetric flow, we can use Euler's equation in cylindrical coordinates:

$$
\begin{aligned}
& \frac{\partial v_{r}}{\partial t}+v_{r} \frac{\partial v_{r}}{\partial r}-\frac{v_{\varphi}^{2}}{r}=-\frac{1}{\rho} \frac{\partial p}{\partial r}, \\
& \frac{\partial v_{\varphi}}{\partial t}+v_{r} \frac{\partial v_{\varphi}}{\partial r}+\frac{v_{r} v_{\varphi}}{r}=0, \\
& \frac{1}{r} \frac{\partial\left(r v_{r}\right)}{\partial r}=0 .
\end{aligned}
$$

The last two equations (6) are satisfied by (1) if $\Gamma$ is constant. Substituting (1) into the first equation (6), and integrating from $r$ to $R$, we obtain

$$
\frac{\mathrm{d} Q}{\mathrm{~d} t} \ln \frac{R}{r}-\frac{Q^{2}+\Gamma^{2}}{2}\left(\frac{1}{r^{2}}-\frac{1}{R^{2}}\right)=\frac{p_{r}-p_{R}}{\rho},
$$


where $p_{r}$ is the gas pressure in the cavity and $p_{R}=p(R)$ is the cylinder wall pressure.

After writing (7) for both cylinders, subtracting and taking into account (5), we get

$$
\frac{\mathrm{d} Q}{\mathrm{~d} t} \ln \frac{R^{2}}{r_{1} r_{2}}-\frac{Q^{2}+\Gamma^{2}}{2}\left(\frac{1}{r_{1}^{2}}-\frac{1}{r_{2}^{2}}\right)+\frac{p_{R_{1}}-p_{R_{2}}}{\rho}=\frac{p_{r_{1}}-p_{r_{2}}}{\rho} .
$$

The pressures $p_{R_{1}}$ and $p_{R_{2}}$ are not equal; their difference is the available head for hydromotors. We characterize it approximately by the standard load coefficient $\zeta$, which is assumed to be a constant, related to the pressure drop $\Delta p$ across the hydromotor by

$$
\Delta p=\frac{1}{2} \zeta\left(\rho / R^{2}\right) Q|Q| \text {. }
$$

Now we choose the chamber radius $R$ as a length scale, the tangential velocity at the chamber entrance $V=v_{\varphi}(R)$ (so that $\Gamma=R V$ ) as the velocity scale, and nondimensionalize the variables. The main nondimensional variable $y(t)$ is introduced by

$$
r_{1}^{2}=R^{2}(\sigma+y), \quad r_{2}^{2}=R^{2}(\sigma-y),
$$

where $y$ can be considered as a volumetric deviation from the equilibrium state $(y=0)$. Note that, because of (10), (3) is satisfied identically. The nondimensional time $\tau$ is

$$
\tau=\frac{2 V}{R} t, \quad \text { so } \quad \frac{\mathrm{d}}{\mathrm{d} t}=\frac{2 V}{R} \frac{\mathrm{d}}{\mathrm{d} \tau} .
$$

From (4) and (11)

$$
Q=V R \dot{y}, \quad \text { and } \mathrm{d} Q / \mathrm{d} t=2 V^{2} \ddot{y},
$$

where the dot means differentiation with respect to $\tau$. Substituting (9)-(12) into (8), we obtain

$$
\ddot{y} \ln \frac{1}{\sigma^{2}-y^{2}}+\frac{\dot{y}^{2}+1}{\sigma^{2}-y^{2}} y+\frac{\zeta}{2}|\dot{y}| \dot{y}=\Pi\left(f_{1}-f_{2}\right),
$$

where $f_{1}-f_{2}=\left(p_{r_{1}}-p_{r_{2}}\right) / p_{0}, \Pi=p_{0} / \rho V^{2}$, and $p_{0}$ is the pressure scale, e.g. minimum pressure in the thermodynamical cycle used. Equation (13) is the principal dynamical equation for VLPE. It is similar to Rayleigh's well-known equation for bubble dynamics, but in contrast, (13) has no solutions for unbounded fluid; it does only in bounded cylindrical domain. Equation (13) is not a closed system because its right hand side is still unknown. Thermodynamic analysis is necessary to determine it.

\subsection{Thermodynamics}

From the first law of thermodynamics for ideal gases,

$$
\delta q=c_{v} \mathrm{~d} T+p \mathrm{~d} V,
$$

where $\delta q$ is the heat supply, $c_{v}$ is the specific heat at a constant volume, and $T$ and $V$ are temperature and volume respectively. Also for an ideal gas,

$$
p V=R T,
$$




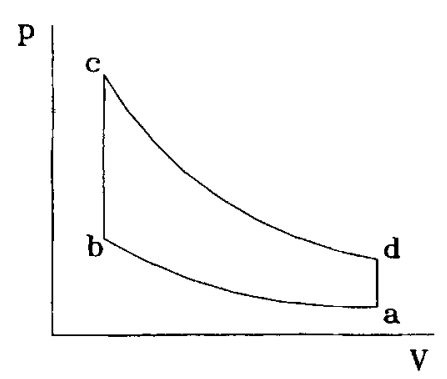

a)

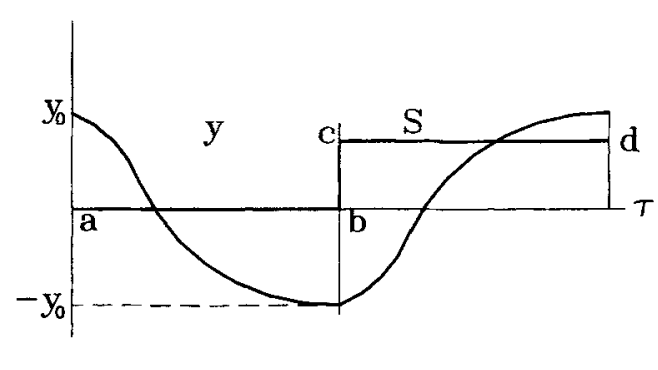

b)

Figure 6. Otto cycle and time-process.

where $R$ is the universal gas constant, i.e. $R=c_{p}-c_{v}=c_{v}(k-1)$ and $k \equiv c_{p} / c_{v}$ is the adiabatic exponent.

Equation (13) has relative volume $y$ and pressure $f$ as dependent variables. Therefore, it is convenient to choose gas volume $V=\pi r_{1}^{2} h=\pi R^{2} h(\sigma+y)$ as the basic thermodynamic variable. Removing $T$ from (14) by using (15) yields

$$
\delta q=(V \mathrm{~d} p+k p \mathrm{~d} V) /(k-1) .
$$

Assuming that the amount of heat $\delta q$ is given, we can write a closed system equation from (14)-(16), in terms of entropy $S$ (using $\delta q=T \mathrm{~d} S$ for a reversible process),

$$
\frac{p}{p_{0}}=\left(\frac{V_{0}}{V}\right)^{k} \exp \left(\frac{k-1}{R} S\right)
$$

where $V_{0}$ is the volume at the pressure $p=p_{0}$ and $S_{0}$ is taken to be zero for convenience.

For a cyclic working engine, the function $S(t)$ must be a periodic function of time. The specific form of $S(t)$ depends on the way heat is supplied, i.e. on the thermodynamic cycle. Most conventional engines operate on the Otto cycle, where heat addition begins during the phase when the piston is near its top dead centre (TDC). At this moment, the volume is near the minimum and combustion occurs very rapidly. In our vortex engine, this phase corresponds to the minimum cavity radius when the radial velocity is zero.

Let us consider, for example, an ideal Otto cycle, consisting of two adiabatic and two isochoric processes (figure 6a). Let this process begin from position $a$ with the maximum volume, when $y=y_{0}$. The adiabatic compression takes place from $a$ to $b$ with $S=0$. Then at the minimum volume (position $b$ ), the system suddenly absorbs heat and goes to position $c$. The entropy increases by a jump, after which there is an adiabatic expansion from $c$ to $d$ and then exhaust from $d$ to $a$. The corresponding time-process is shown in figure $6 \mathrm{~b}$ (the curve denotes the periodic condition (21), and the other cylinder is $90^{\circ}$ out-of-phase with this one).

For the Otto cycle the value $f_{1}-f_{2}$ from (13) can be found as

$$
f_{1}-f_{2}=\left(\frac{\sigma+y_{0}}{\sigma+y}\right)^{k}-\left(\frac{\sigma+y_{0}}{\sigma-y}\right)^{k} \exp \left(\frac{k-1}{R} S\right)
$$


where $S=$ const. during the adiabatic half-period. Note that $\sigma$ and $y_{0}$ determine the compression ratio $\epsilon$

$$
\epsilon=\left(\sigma+y_{0}\right) /\left(\sigma-y_{0}\right)
$$

The Otto cycle is chosen as an example only; real cycles are smoother. Equation (13), which governs the dynamics of our vortex liquid piston engine, has three parameters: $\sigma$ (the total gas/cylinder volume ratio), $\zeta$ (the load coefficient), and $\Pi$ (the pressure parameter). Two additional parameters, $S$ (the heat parameter) and $y_{0}$ (the magnitude of oscillations), are contained in (18). Thus the system is characterized by five independent parameters, which are large for a complete parametric analysis. Some physical conditions will be used to reduce the number of independent parameters.

\subsection{Physical conditions}

Periodicity condition: Equations (13) and (18) form a closed autonomous system with the following initial conditions

$$
y(0)=y_{0} \text { and } \dot{y}(0)=0,
$$

where the initial time $\tau=0$ is chosen at maximum $y(\tau)=y_{0}$. The value $y_{0}$ in (18) is not arbitrary but has to be determined from the periodic condition. We assume that in both cylinders, the processes are identical but only shifted by half a period $\frac{1}{2} \tau_{c}$, i.e.,

$$
r_{2}\left(\tau+\frac{1}{2} \tau_{c}\right)=r_{1}(\tau)
$$

Taking into account (10), we obtain

$$
y\left(\tau+\frac{1}{2} \tau_{c}\right)=-y(\tau) .
$$

Then from (21), we get

$$
y\left(\frac{1}{2} \tau_{c}\right)=-y_{0} \quad \text { and } \quad \dot{y}\left(\frac{1}{2} \tau_{c}\right)=0 .
$$

According to (21), (13) should be solved only in the interval $0 \leq \tau \leq \frac{1}{2} \tau_{c}$ but the value $y_{0}$ must satisfy (22); this can be achieved by an iterative procedure. It is the principal difference in comparison with the conventional internal combustion engine, where the piston amplitude and compression ratio are fixed.

Combustion condition: Let us consider an Otto cycle consisting of the two adiabatic curves ( $\mathrm{ab}$ and $\mathrm{cd}$ ) and two isochores ( $\mathrm{bc}$ and da) shown in figure 6a. Heat is received along $\mathrm{bc}$ and is rejected along da. Along the process bc, $\delta q=c_{v} \mathrm{~d} T$ and $q=c_{v}\left(T_{c}-T_{b}\right)$; thus

$$
q=q_{f} /\left(1+n_{0} \alpha\right)
$$

where $q_{f}$ is enthalpy of reaction, $\alpha$ is the relative air/fuel ratio, and $n_{0}$ is the stoichiometric (or chemically correct or theoretical) air/fuel ratio. Thus $n_{0} \alpha$ gives the actual air/fuel ratio. Since $q=c_{v}\left(T_{c}-T_{b}\right)$ we have

$$
T_{c}-T_{b}=q_{f} /\left[c_{v}\left(1+n_{0} \alpha\right)\right]
$$


On the other hand, for an isochoric process

$$
S_{c}-S_{b}=c_{v} \ln \left(T_{c} / T_{b}\right)=c_{v} \ln E,
$$

where

$$
E=1+\frac{q_{f}}{c_{v}\left(n_{0} \alpha+1\right) T_{0} \epsilon^{k-1}}=1+\frac{E_{0}}{\epsilon^{k-1}} .
$$

For a typical hydrocarbon fuel, $q_{f} \cong 20,000 \mathrm{Btu} / \mathrm{lb} \cong 46,500 \mathrm{~kJ} / \mathrm{kg}$. For typical cases using hydrocarbons, $n_{0}$ is about $15 \mathrm{~kg}$ air $/ \mathrm{kg}$ fuel, $T_{0}=300 \mathrm{~K}$, and $\alpha=1.25$. So $E_{0} \cong 11$, and for an Otto cycle $E=1+11 \epsilon^{1-k}$. We can rewrite (18) in terms of $E$ :

$$
f_{1}-f_{2}=\left(\frac{\sigma+y_{0}}{\sigma+y}\right)^{k}-\left(\frac{\sigma+y_{0}}{\sigma-y}\right)^{k} E,
$$

where $E$ is given by (26), and (13) can be solved accordingly.

Kinematic condition: The flow in a vortex chamber is created by a special device, a swirler, which in principle can move or be at rest. In the case of a porous rotating cylinder, the flow in the vortex chamber is characterized by two independent functions $Q(t)$ and $\Gamma(t)$. Of course, in case of a fixed swirler, $Q$ and $\Gamma$ are not independent. This can be illustrated by a steady flow in a vortex chamber. Let the swirler contain a number of narrow tangential slits. The flow rate into the chamber can be approximated as $Q_{v}=n b h V$, and according to (2) we have

$$
Q=(n b / 2 \pi) V,
$$

where $n$ is the number of slits, each of width $b$ (figure 7).

On the other hand, according to (1),

$$
Q=R\left|v_{r}(R)\right|,
$$

where $v_{r}(R)$ is the azimuthally averaged radial velocity. From (1) it follows that the design parameter,

$$
\theta=\frac{Q}{\Gamma}=\frac{\left|v_{r}\right|}{v_{\varphi}}=\frac{n b}{2 \pi R},
$$

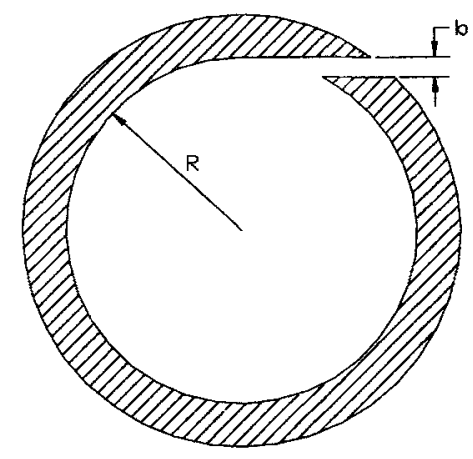

Figure 7. Tangential slit. 


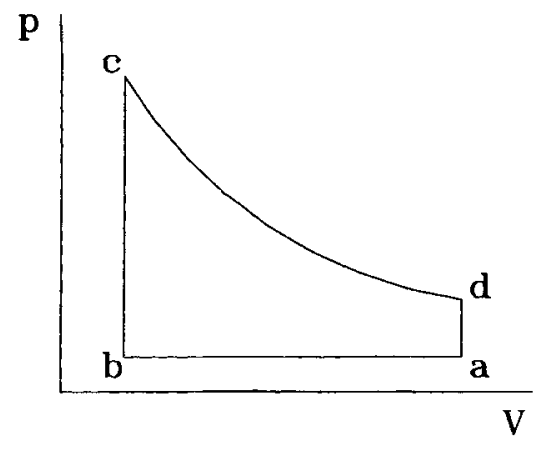

Figure 8. Semi-Otto cycle.

is a purely geometric characteristic of the vortex chamber which relates $Q$ to $\Gamma$. Equation (30) shows that, once a design is chosen, $\theta$ should be a constant. For unsteady flow, (28) is no longer valid, but (29) is. For example, at the instant when $Q=0, v_{r}$ is also zero, but $\Gamma$ is obviously not because of vortex inertia (i.e. even without a flow rate, the liquid continues to rotate). For an inviscid liquid, such a flow will continue forever with maximum $\Gamma$. For real flow, if the viscous time $\left(\sim R^{2} / v\right)$ is much larger than the period of oscillation, we can assume that (30) is correct for the maximum $Q$, and thus $\Gamma=Q_{\max } / \theta$.

Using (30) and (12) we find the important relationship between system dynamics and design parameter $\theta$

$$
|\dot{y}|_{\max }=(n b / 2 \pi R)=\theta .
$$

This equation is the condition for the swirler to be at rest. The derivation of (31) is speculative and needs to be verified by experiment with unsteady separation process. Equation (29) assumes an axisymmetric configuration of the entrances. So the number of entrances $(n)$ should be relatively large.

Semi-Otto cycle: Experimental realization of the Otto cycle is difficult because we need a very high maximum pressure in order to use a realistic compression ratio; our goal is only to prove the concept, i.e. to establish the possibility of oscillating stable cavity generation. In order to model the cavity with comparable liquid velocities, we are going to use a semi-Otto cycle (as shown in figure 8) in our subscale "cold" experimental model (using compressed air supply) because it operates at lower pressures than the Otto cycle at the same compression ratio.

In our experiments the parameter $E$ is not meaningful, therefore we introduce a pressure ratio parameter $\chi$ which allows us to consider both cycles:

$$
\chi=p_{\max } / p_{\min }=p_{c} / p_{a} .
$$

The values $E$ and $\chi$ are related. For the Otto cycle,

$$
\chi=\epsilon^{k} E \text {. }
$$

Introducing $\chi$ into (27), we obtain

$$
f_{1}-f_{2}=\left(\frac{\sigma+y_{0}}{\sigma+y}\right)^{k}-\chi\left(\frac{\sigma-y_{0}}{\sigma+y}\right)^{k} .
$$


For the semi-Otto cycle we have

$$
f_{1}-f_{2}=1-\chi\left(\frac{\sigma-y_{0}}{\sigma-y}\right)^{k} \text {. }
$$

\subsection{Energy equation and operating characteristics}

We found that (13) needs to be solved for half a period $0 \leq \tau \leq \tau_{c} / 2$ only, where $\dot{y}(\tau) \leq 0$ and $(\zeta / 2)|\dot{y}| \dot{y}=-(\zeta / 2) \dot{y}^{2}$. Then it can be shown that (13) can be written in the form

$$
\frac{1}{2} \frac{\mathrm{d}}{\mathrm{d} y}\left[\left(\dot{y}^{2}+1\right) \ln \frac{1}{\sigma^{2}-y^{2}}\right]=\frac{\zeta}{2} \dot{y}^{2}-\Pi\left(f_{2}-f_{1}\right) .
$$

Integrating (36) for the half-period of oscillation between $y=-y_{0}$ and $y=y_{0}$, we obtain the energy equation

$$
\frac{\zeta}{2} \int_{-y_{0}}^{y_{0}} \dot{y}^{2} \mathrm{~d} y=\Pi \int_{-y_{0}}^{y_{0}}\left(f_{2}-f_{1}\right) \mathrm{d} y .
$$

Equation (37) states that inertial features do not participate directly in the energy balance. The right-hand side of (37) is the work (during a thermodynamic cycle) and the left-hand side is the energy consumption. Equation (37) helps to deduce the power $W$ of the engine. The general expression for $W$ is

$$
W=2 \pi R^{2} h \rho V^{2} f \Pi \int_{-y_{0}}^{y_{0}}\left(f_{1}-f_{2}\right) \mathrm{d} y,
$$

where $f$ is the frequency,

$$
f=2 V / R \tau_{c} \text {. }
$$

For an Otto cycle, a simple calculation gives

$$
\int_{-y_{0}}^{y_{0}}\left(f_{1}-f_{2}\right) \mathrm{d} y=\frac{\sigma+y_{0}}{k-1} \eta E_{0}
$$

where $\eta=1-\epsilon^{1-k}$ is the well-known efficiency for Otto cycle (Faires \& Virgil 1970), $\epsilon$ and $E_{0}$ are given by (19) and (26). For the semi-Otto cycle, using (27), we have

$$
\int_{-y_{0}}^{y_{0}}\left(f_{1}-f_{2}\right) \mathrm{d} y=\chi \frac{\sigma-y_{0}}{k-1}\left(1-\epsilon^{1-k}\right)-2 y_{0} \text {. }
$$

It can be shown that the efficiency for this semi-Otto cycle is

$$
\eta=1-\frac{k(\epsilon-1)+\chi \epsilon^{1-k}-\epsilon}{\chi-1} .
$$

The efficiency $\eta$ of the Otto cycle depends on the compression ratio $\epsilon$ alone while that of the semi-Otto cycle depends on both $\epsilon$ and $\chi$. Note that at a fixed $\chi$, the efficiency (42) has a maximum at $\epsilon=\chi^{1 / k}$, where $\partial \eta / \partial \epsilon=0$. Then

$$
\eta_{\max }=1-\frac{k\left(\chi^{(1 / k)}-1\right)}{\chi-1} \text { or } \quad \eta_{\max }=1-\frac{k(\epsilon-1)}{\epsilon^{k}-1} .
$$

Figure 9 shows a comparison of this efficiency with that of the Otto cycle with $k=1.4$.

Note that for any given compression ratio $\epsilon, \eta_{\mathrm{Otto}}>\eta_{\max }$. However, for a fixed pressure ratio $\chi$ in the working range, $\eta_{\max }>\eta_{\text {Otto }}$. 


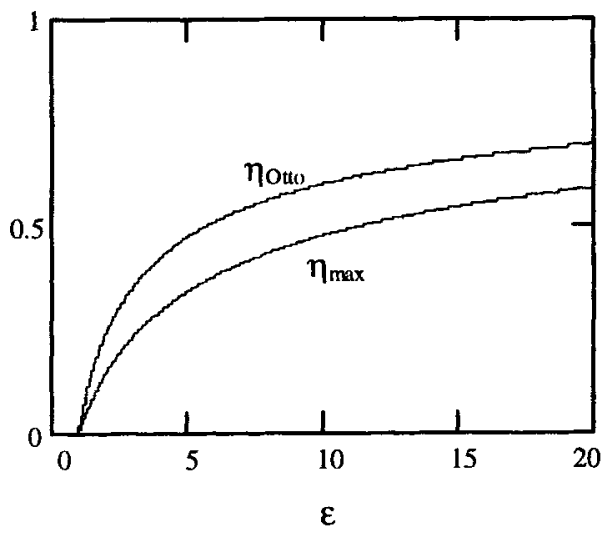

Figure 9. Comparison of efficiency.

\subsection{Free oscillations}

Unlike the conventional solid piston engines where the piston displacements are fixed, the VLPE has a variable displacement which depends on system parameters. In order to achieve the maximum oscillating amplitude (which gives maximum power and efficiency), it is very important to know the eigenfrequency of the VLPE. This may also benefit the creation of a "shrinking engine" (Popular mechanics 1995) whose cylinder size can be changed corresponding to load to reduce energy losses.

Small oscillations: The eigenfrequency for small free oscillations can be obtained from (13) by assuming that both $y$ and $\dot{y}$ are small; that is

$$
\ddot{y} \ln \frac{1}{\sigma^{2}}+\frac{1}{\sigma^{2}} y=\Pi\left(f_{1}-f_{2}\right) .
$$

For an adiabatic process, by letting $S=0$ in (18), we have for the right-hand side of (44),

$$
\Pi\left(f_{1}-f_{2}\right)=\Pi\left[\left(\frac{V_{0}}{V_{1}}\right)^{k}-\left(\frac{V_{0}}{V_{2}}\right)^{k}\right]=\Pi\left[\left(\frac{\sigma+y_{0}}{\sigma+y}\right)^{k}-\left(\frac{\sigma+y_{0}}{\sigma-y}\right)^{k}\right] .
$$

When $y \ll 1$ and $y_{0} \ll 1$,

$$
\left(\frac{\sigma+y_{0}}{\sigma+y}\right)^{k}=1-\frac{k}{\sigma} y+\cdots \quad \text { and }\left(\frac{\sigma+y_{0}}{\sigma-y}\right)^{k}=1+\frac{k}{\sigma} y+\cdots
$$

Using these expansions in (44) and (45), the equation for small free oscillations becomes

$$
\ddot{y} \ln \frac{1}{\sigma^{2}}+\left(\frac{1}{\sigma^{2}}+\frac{2 k \Pi}{\sigma}\right) y=0,
$$

and the eigenfrequency is

$$
\omega_{0}=\left(\frac{1+2 k \Pi \sigma}{\sigma^{2} \ln \left(1 / \sigma^{2}\right)}\right)^{1 / 2}
$$




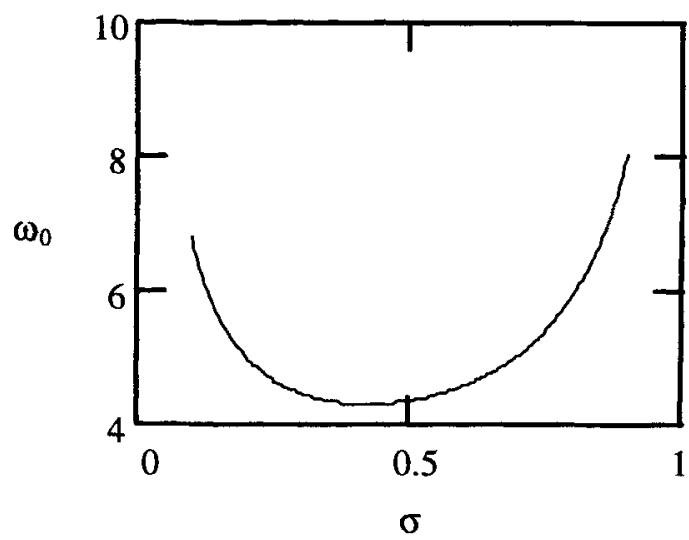

Figure 10. $\omega_{0}$ as a function of $\sigma$ with fixed $\Pi$.

(48) shows that $\omega_{0}$ has a minimum value with respect to $\sigma$. This value is achieved when $\sigma$ satisfies the following equation

$$
(1+\ln \sigma)(1+k \Pi \sigma)=\frac{1}{2} .
$$

Figure 10 depicts $\omega_{0}$ as a function of $\sigma$ with $k=1.4$ (for air) and nondimensional pressure $\Pi=4.0$ (for $p_{0}=10^{5} \mathrm{~Pa}$ and $V=5 \mathrm{~m} / \mathrm{s}, \Pi=p_{0} / \rho V^{2}$ ). From this figure, we can see that $\omega_{0}$ has a minimum at $\sigma=\sigma_{\min }$. This unique feature will be explained later together with figure 14 below. Figure 11 shows that $\sigma_{\min }$ has its largest value, $\sigma_{\min }=(1 / e)^{1 / 2} \approx 0.6065$, at $\Pi=0$ and decreases with increasing $\Pi$. Figure 12 shows how the minimum frequency changes with $\Pi$.

Nonlinear free oscillations: Let us consider (36) with $\zeta=\Pi=0$. In this case, (36) can be integrated to give

$$
\left(\dot{y}^{2}+1\right) \ln \frac{1}{\sigma^{2}-y^{2}}=\text { const } .
$$

The constant can be found from the condition $\dot{y}\left(y_{0}\right)=0$, and solving for $\dot{y}$ gives

$$
\dot{y}=\sqrt{\frac{\ln \left(\sigma^{2}-y_{0}^{2}\right)}{\ln \left(\sigma^{2}-y^{2}\right)}-1} .
$$

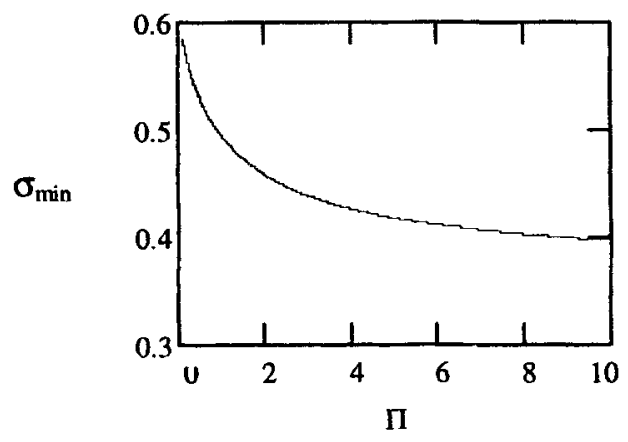

Figure 11. $\sigma_{\min }$ as a function of $\Pi$. 


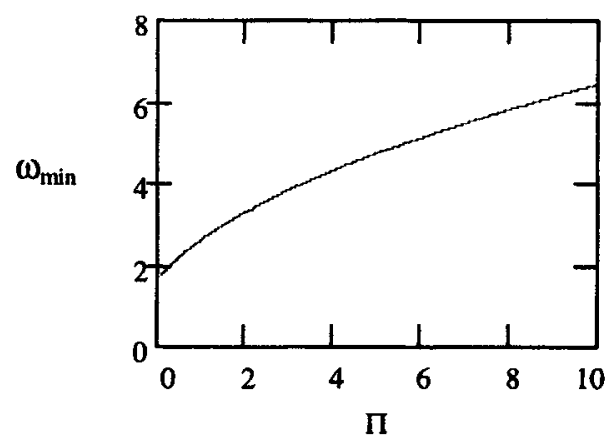

Figure 12. $\omega_{\min }$ as a function of $\Pi$.

Integrating (50) for half-period $\tau_{c} / 2$ we have

$$
\tau_{c}=4 \int_{0}^{y_{0}}\left(\frac{\ln \left(\sigma^{2}-y_{0}^{2}\right)}{\ln \left(\sigma^{2}-y^{2}\right)}-1\right)^{-1 / 2} \mathrm{~d} y .
$$

Defining the eigenfrequency $f_{c}$ as

$$
f_{c}=1 / \tau_{c}
$$

from (39) we have

$$
f=(2 V / R) f_{c} \text {. }
$$

Figure 13 shows $f_{c}$ as a function of $y_{0}$ for the cases $\sigma=0.4,0.5$, and 0.6 . We see that for the typical $\sigma$ range, eigenfrequency increases with increasing amount of fluid in the system as well as with oscillating amplitude $y_{0}$. Both these features are the opposite of those of an ordinary oscillating system like a U-tube where increasing amount of liquid decreases the eigenfrequency and oscillating amplitude decreases with increase in eigenfrequency. These features are useful for a real engine, because with the increase of engine frequency and power, the oscillating amplitude increases and so does the efficiency, (19).

Figure 14 shows $f_{c}$ as a function of $\sigma$ for the case $y_{0}=0.15$. The eigenfrequency has a minimum with respect to the gas/liquid ratio $\sigma$ for finite amplitude oscillation. This somewhat unusual non-monotonic behaviour of the eigenfrequency is related to the strong dependence of centrifugal force $\rho v_{\varphi}^{2} / r$ on $r$ and can be explained by making analogy with

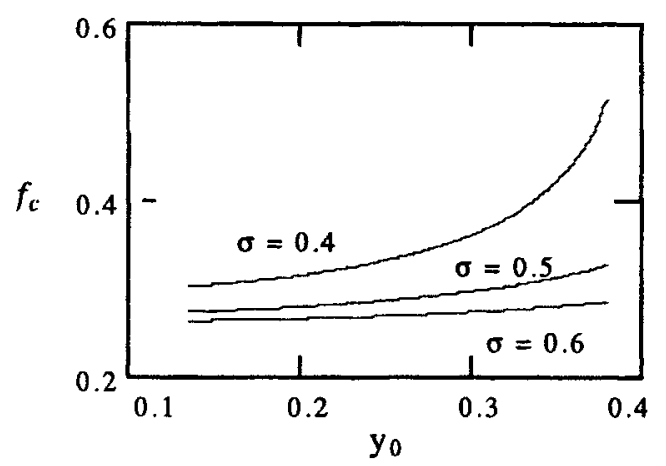

Figure 13. $f_{c}$ as a function of $y_{0}$ with fixed $\sigma$. 


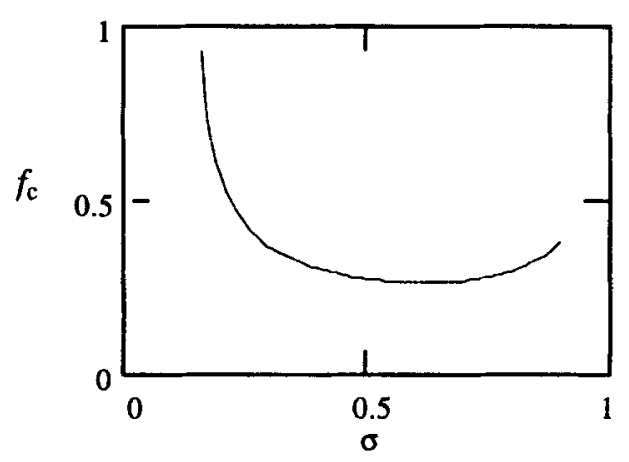

Figure 14. $f_{c}$ as a function of $\sigma$ with fixed $y_{0}$.

a U-tube system. For the U-tube, the eigenfrequency is $\omega=(g / l)^{1 / 2}$, where $l$ is the height. For VLPE, when $\sigma$ is small, we have larger amount of liquid such that $r$ is small. Then the centrifugal force $\rho v_{\varphi}^{2} / r$ tends to infinity as $\sigma$ tends to zero. This is equivalent to a larger $g$ case for the U-tube. Therefore, the eigenfrequency is very high for small $\sigma$. On the other hand, when $\sigma$ is large, we have small amount of liquid such that the corresponding $l$ is small. Between these two extremes there must be a minimum.

\subsection{Stability criterion}

The stability problem is very complicated in the general case, because the corresponding linearized hydrodynamic equation has time-dependent coefficients, and Floquet theory must be used. Therefore, we prefer to investigate the general stability problem by experiment. However, a simple criterion $S_{t}$, necessary for stability, can be found from elementary considerations:

$$
S_{t}=\min \left(g_{c} / g_{r}\right)=\min 1 /\left[2(\sigma+y) \ddot{y}-\dot{y}^{2}\right],
$$

where $g_{c}\left(=v_{\varphi}^{2} / r\right)$ is the centrifugal acceleration and $g_{r}$ is the radial acceleration. If the maximum is achieved at the moment of maximum compression, (54) is slightly simplified $(\dot{y}=0)$. Clearly, $g_{c}$ must be greater than $g_{r}$ or else the cavity will collapse; hence, the minimal $S_{t}=1$. We therefore use $S_{t}>1$ as the stability criterion. The larger the value of $S_{t}$, the more stable the rotating flow. Another related condition is $g_{c} \gg g$, where $g$ is gravity.

\subsection{Variable circulation}

The previous formulation was based on the assumption that $\Gamma=$ const. But how do we choose this constant? It clearly depends on flow rate $Q$; in particular, if $Q \equiv 0$ then $\Gamma \equiv 0$. For a steady flow, $\Gamma=-\theta Q$, where $\theta=v_{\phi} / v_{r}=\tan \alpha=$ const is a swirl parameter and $\alpha$ is the blade angle of the swirler device. This follows from the Kutta condition (i.e. no separation) for inviscid flow past blades. If a process is unsteady, the direct application of the Kutta condition should give

$$
\Gamma(t)=-\theta Q(t), \quad Q(t)<0 .
$$


But the validity of the Kutta condition for unsteady flow is doubtful because of possible flow separation. Nevertheless, in this section, we consider (55) to be valid. For such a situation, $\Gamma$ is not a constant and depends not only on $t$, but also on $r$. We use an equation for $\Gamma$

$$
\frac{\partial \Gamma}{\partial t}+v_{r} \frac{\partial \Gamma}{\partial r}=0 ; \quad v_{r}=\frac{Q}{r} .
$$

Although $\Gamma=$ const. is a solution of (56), it does not satisfy (55). (56) can be solved by the method of characteristics. Let us introduce the variables $z_{i}(\tau)$ and $s(t)$, such that

$$
z_{i}(\tau)=\frac{r_{i}^{2}(\tau)}{R^{2}}, \quad i=1,2 ; \quad \mathrm{d} s=\frac{2 Q}{R^{2}} \mathrm{~d} t
$$

Note that if $Q=$ const. $=R V$, we have $s=\tau$.

From (10),

$$
r_{1}^{2}+r_{2}^{2}=2 R^{2} \sigma
$$

Using the new variables, (56) can be rewritten as

$$
\frac{1}{R^{2}} \frac{\partial \Gamma}{\partial s}+\frac{\partial \Gamma}{\partial r^{2}}=0
$$

The general solution of this equation is

$$
\Gamma(r, \tau)=F\left(r^{2}-R^{2} s\right),
$$

where $F$ is an arbitrary function which can be found from the boundary condition at $r=R$. The boundary condition (55) then becomes

$$
F\left(R^{2}(1-s)\right)=-\theta Q(\tau)
$$

If $s$ is known, we can determine the function $F$ using the inverse function $\tau=\tau(s)$.

To derive the equations of motion, we assume that at the initial time $\tau=0$ both cylinders are filled with equal amounts of liquid, rotating with $\Gamma=\Gamma_{0}=$ const. Once the motion starts, a part of the liquid flows into one cylinder from the other according to (55). Under such conditions, liquid in the first cylinder still has circulation $\Gamma_{0}$. Let $b_{1}$ be the radius of the boundary between this liquid and the remainder liquid having another circulation.

Thus the circulation distribution is

$$
\Gamma(r, t)= \begin{cases}\Gamma_{0}, & r_{1} \leq r \leq b_{1}, \\ F\left(r^{2}-R^{2} s\right), & b_{1}<r \leq R .\end{cases}
$$

Mass balance gives

$$
\pi\left(R^{2}-b_{1}^{2}\right)=\pi\left(R^{2}-r_{1}^{2}\right)-\pi\left(R^{2}-r_{1_{\max }}^{2}\right) .
$$


Therefore,

$$
\begin{aligned}
& b_{1}^{2}=R^{2}\left(1+z-z_{\max }\right), \quad z_{\max }=\max _{0 \leq t^{\prime} \leq t} z\left(t^{\prime}\right), \text { and } \\
& \int_{r_{1}}^{R} \frac{\Gamma_{0}^{2}}{r^{3}} \mathrm{~d} r=2 \pi^{2} c^{2} \gamma_{0}^{2}\left(\frac{1}{z}-\frac{1}{1+z-z_{\max }}\right) .
\end{aligned}
$$

Consider the integral

$$
\int_{r_{1}}^{b_{1}} \frac{\Gamma_{0}^{2}}{r^{3}} \mathrm{~d} r+\int_{b_{1}}^{R} \frac{F^{2}\left(r^{2}-R^{2} z\right)}{r^{3}} \mathrm{~d} r .
$$

The first part of (62) is given by (61). To calculate the second part, we introduce variables

$$
z^{\prime}=1-r^{2} / R^{2} ; \quad z_{*}=1-b_{1}^{2} / R^{2} ; \quad \xi=z+z^{\prime}
$$

Using (59), the second part of (62) becomes

$$
\begin{aligned}
& \int_{b_{1}}^{R} \frac{F^{2}\left(r^{2}-R^{2} z\right)}{r^{3}} \mathrm{~d} r=\int_{0}^{z_{*}} \frac{F^{2}\left(R^{2}\left(1-z-z^{\prime}\right)\right)}{2 R^{2}\left(1-z^{\prime}\right)^{2}} \mathrm{~d} z^{\prime} \\
& \quad=\int_{z}^{z_{\max }} \frac{F^{2}\left(R^{2}(1-\xi)\right)}{2 R^{2}(1+z-\xi)^{2}}=\frac{\theta^{2}}{2 R^{2}} \int_{z}^{z_{\max }} \frac{Q^{2}(\tau(\xi))}{(1+z-\xi)^{2}} \mathrm{~d} \xi \\
& \quad=\frac{\theta^{2} C^{2}}{8} \int_{z(\tau)}^{z_{\max }(\tau)} \frac{\dot{z}^{2}(\sigma)}{\left[1+z(\tau)-z^{\prime}\right]^{2}} \mathrm{~d} z^{\prime} .
\end{aligned}
$$

In order to integrate equation (63), it is sufficient to know the dependence of $z(z)$ for all previous time. In the same way, we can obtain the corresponding relation for the second cylinder by integrating (2) from $r_{2}$ to $R$ and taking into account the boundary conditions determined by the swirler at the moment of inflow. Thus, we obtain

$$
\Gamma_{2}(R, t)=F_{2}\left(R^{2}(1-s)\right)=\theta Q(\tau(s)), \quad Q>0\left(Q_{2}=-Q\right) .
$$

The result is slightly different from (8).

Finally we obtain the generalization of (13):

$$
\begin{aligned}
\ddot{z} & \ln \frac{1}{z(2 \sigma-z)}+\left(1+\frac{\dot{z}^{2}}{4}\right)\left(\frac{1}{2 \sigma-z}-\frac{1}{z}\right)+\frac{1}{1+z-z_{\max }} \\
& -\frac{1}{1+z_{\min }-z}+\frac{\theta^{2}}{4}\left[\int_{z_{\min }}^{z} \frac{\dot{z}^{2}\left(z^{\prime}\right)}{\left(1-z+z^{\prime}\right)^{2}} \mathrm{~d} z^{\prime}\right. \\
& \left.-\int_{z}^{z_{\max }} \frac{\dot{z}^{2}\left(z^{\prime}\right)}{\left(1-z+z^{\prime}\right)^{2}} \mathrm{~d} z^{\prime}+\zeta \frac{\dot{z}^{2}}{4} \operatorname{sign} \dot{z}-4 \sigma \frac{\sigma-z}{z(2 \sigma-z)}\right]=0,
\end{aligned}
$$

where $z_{\max }(t)=\max _{0 \leq \tau^{\prime} \leq \tau} z\left(t^{\prime}\right), z_{\min }(t)=\min _{0 \leq \tau^{\prime} \leq \tau} z\left(t^{\prime}\right)$, and $0 \leq z(t) \leq \min (1,2 \sigma)$.

The integro-differential equation (64) determines the evolution $s(\tau)$ for a given variation of the temperature in the cavities.

The initial conditions are

$$
z(0)=z_{0}, \quad \dot{z}(0)=0, \quad z_{\max }(0)=z_{0}, \quad \text { and } \quad z_{\min }(0)=z_{0} .
$$




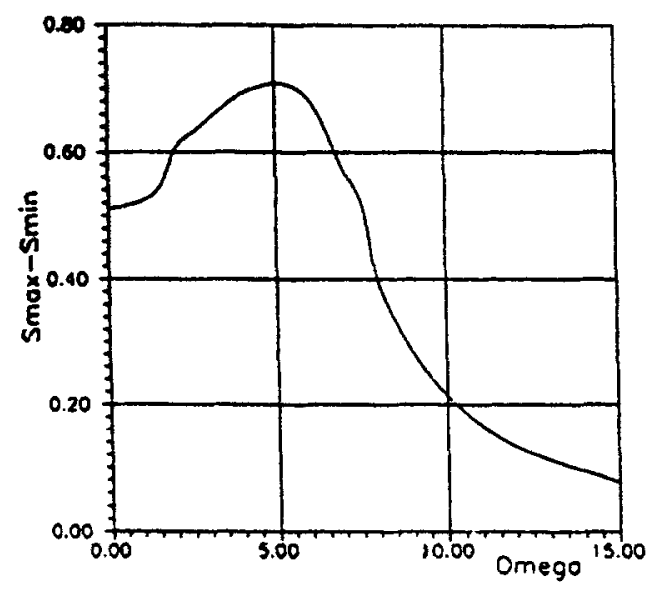

Figure 15. Resonance frequency.

Numerical calculations of the system (64)-(65) were performed using the Runge-Kutta method with adaptive step control. The integrals in (64) were calculated using the technique of nonlocal splines at each step of integration.

Figure 15 shows the dependence of amplitude $\Delta S=S_{\max }-S_{\min }$ for the periodic regime versus the forcing frequency $\omega$. This shows that there is some resonance frequency $\omega^{*}$. The small bumps correspond to $\omega^{*} / 2$ and $3 \omega^{*} / 2$. The solution near $\omega^{*}$ has a minimal transition time to periodic motion. The phase portrait of the transient process for $\omega=\omega^{*}$ is represented in figure 16. The phase trajectories of the transient processes are shown in figure 17 for $\omega>\omega^{*}$ and in figure 18 for $\omega<\omega^{*}$.

Note that these results suggest that we can expect the minimal perturbations of cavity surfaces near resonance. The complex transient process with high-frequency oscillations corresponds to a flow with $\omega \ll \omega^{*}$ (figures 19 and 20).

If $\omega \gg \omega^{*}$, the high-frequency perturbations do not appear but amplitudes $z$ and $z$ grow strongly and exceed amplitudes of periodic regime (figures 21 and 22). This analysis shows that regime with $\omega \approx \omega^{*}$ is preferable.

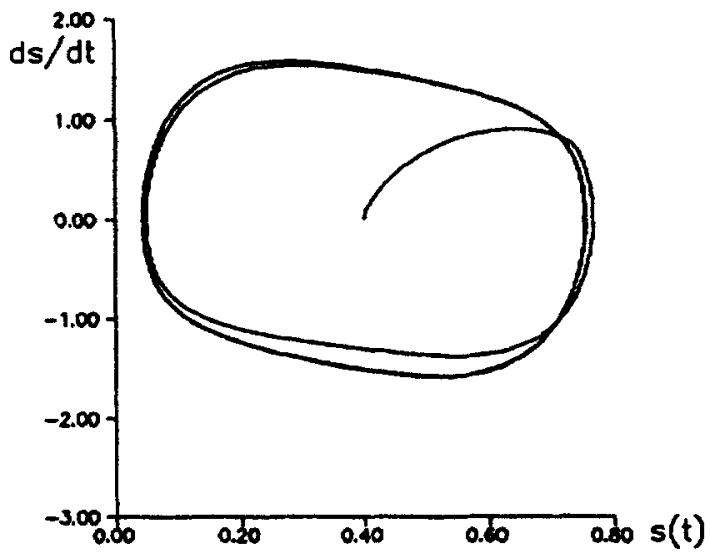

Figure 16. Phase portrait of the transient process for $\omega=\omega$. 


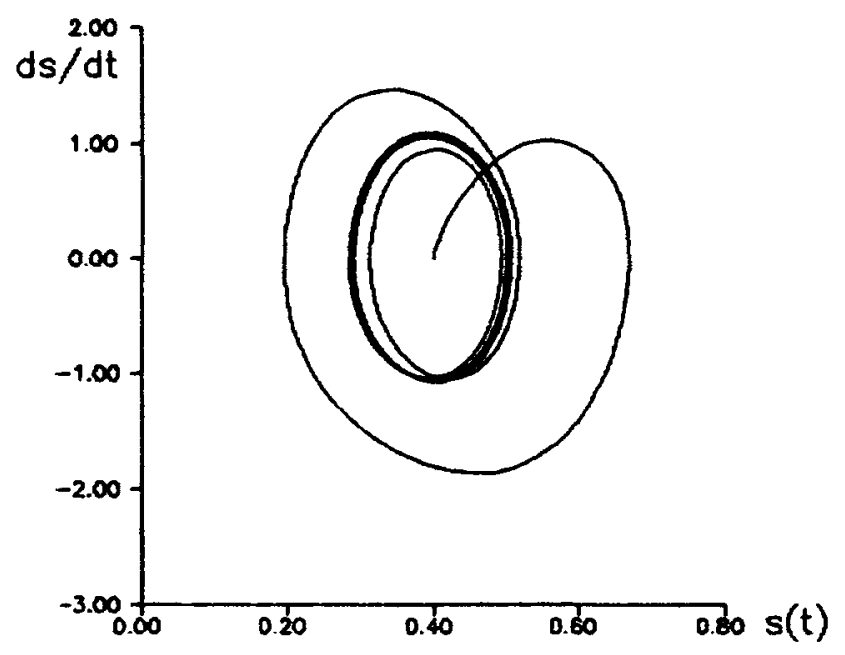

Figure 17. Phase portrait of the transient process for $\omega>\omega^{*}$.

The calculations with $\Gamma=$ const. show that an important difference exists only for small frequencies $\omega \ll \omega^{*}$. If $\omega>\omega^{*}$, the resonance curves practically coincide (the resonance curve for $\Gamma=$ const. is shown in figure 23 by the dashed curve).

The general conclusion is: if the frequency is sufficiently high $\left(\omega>\omega^{*}\right)$, the assumption of $\Gamma=$ const. is reasonable, because, in this case, the transient processes can be considered quasi-stationary processes.

\subsection{The role of viscosity}

Let the flow rate $Q(t)$ be given. The equation for circulation $\Gamma$ is

$$
\frac{\partial \Gamma}{\partial t}+\frac{Q(t)}{r} \frac{\partial \Gamma}{\partial r}=v r \frac{\partial}{\partial r} \frac{1}{r} \frac{\partial \Gamma}{\partial r},
$$

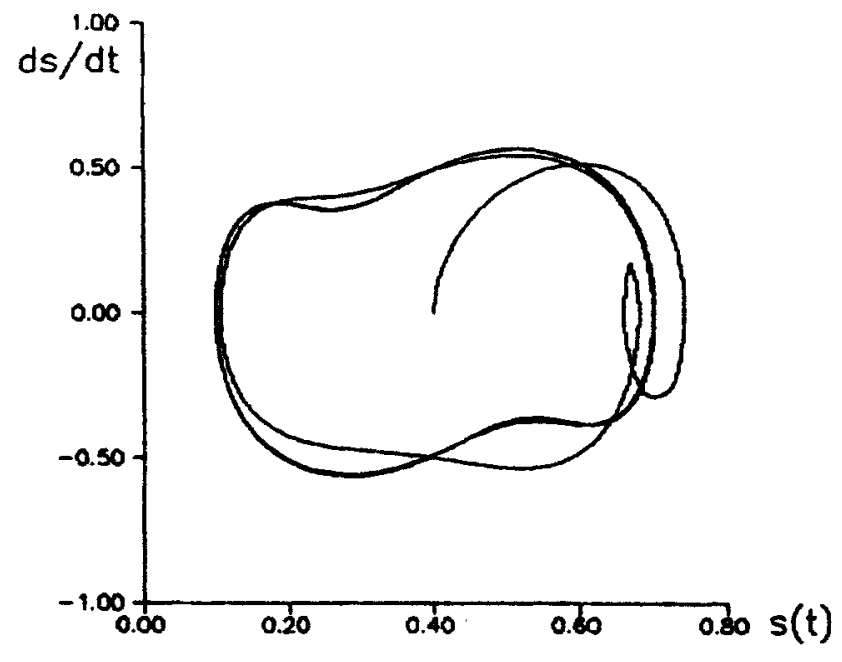

Figure 18. Phase portrait of the transient process for $\omega<\omega^{*}$. 


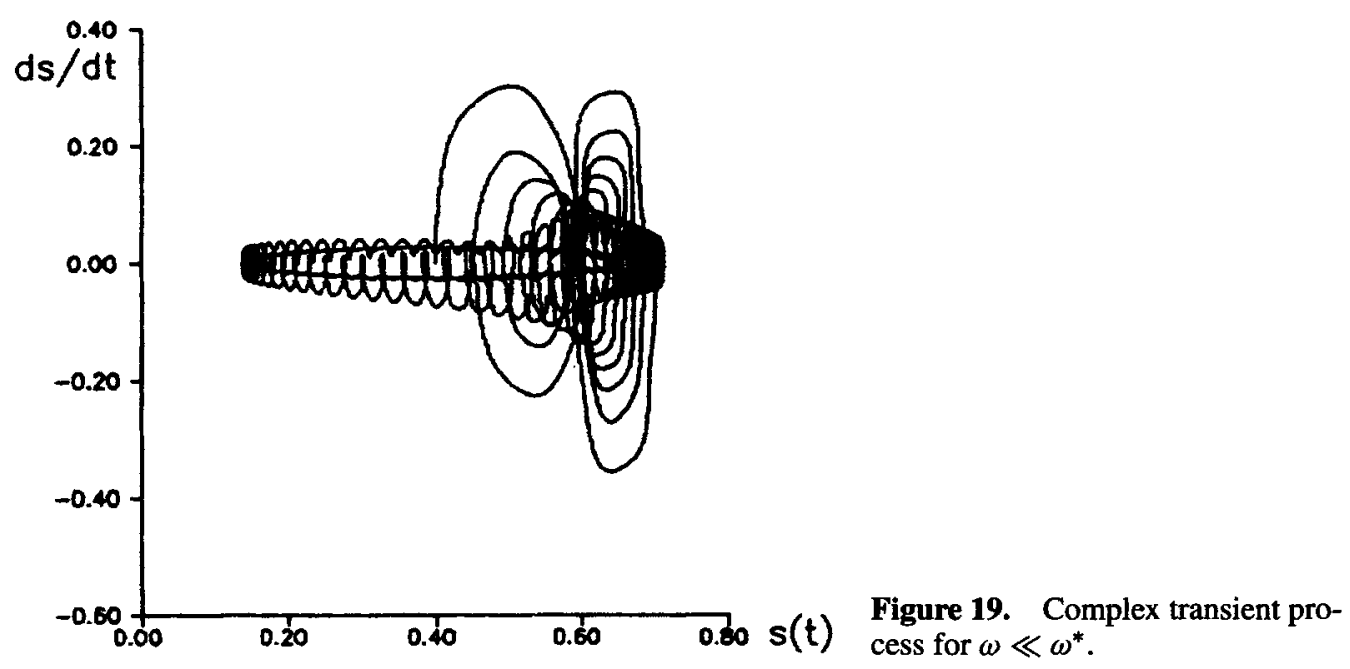

where $v$ is the kinematic viscosity. Assume $r_{1}=r_{0}(1+a \sin \omega t)$, and choose $R$ as the length scale to nondimensionalize $r$. Introducing other variables

$$
\tau=\frac{\nu t}{R^{2}} ; \quad \Omega=\frac{\omega R^{2}}{\nu} ; \quad q=\frac{Q(t)}{\nu} ; \quad \text { and } \quad q_{0}=\Omega r_{0}^{2} a,
$$

we have

$$
Q=R^{2} r_{1} \dot{r}_{1}=R^{2} r_{0}^{2} a \omega(1+a \sin \Omega \tau) \cos \Omega \dot{\tau},
$$

and

$$
q=q_{0}(1+a \sin \Omega \tau) \cos \Omega \tau .
$$

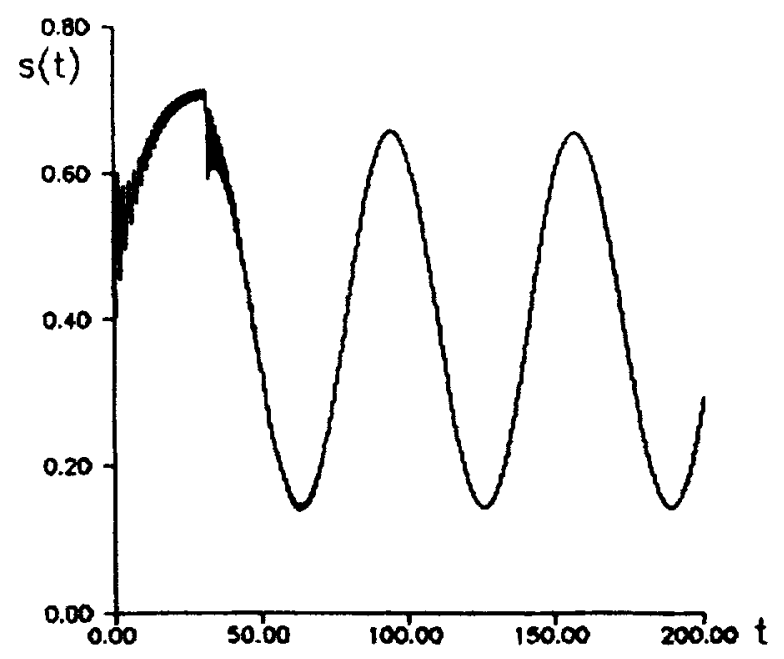

Figure 20. Complex transient process for $\omega \ll \omega^{*}$. 

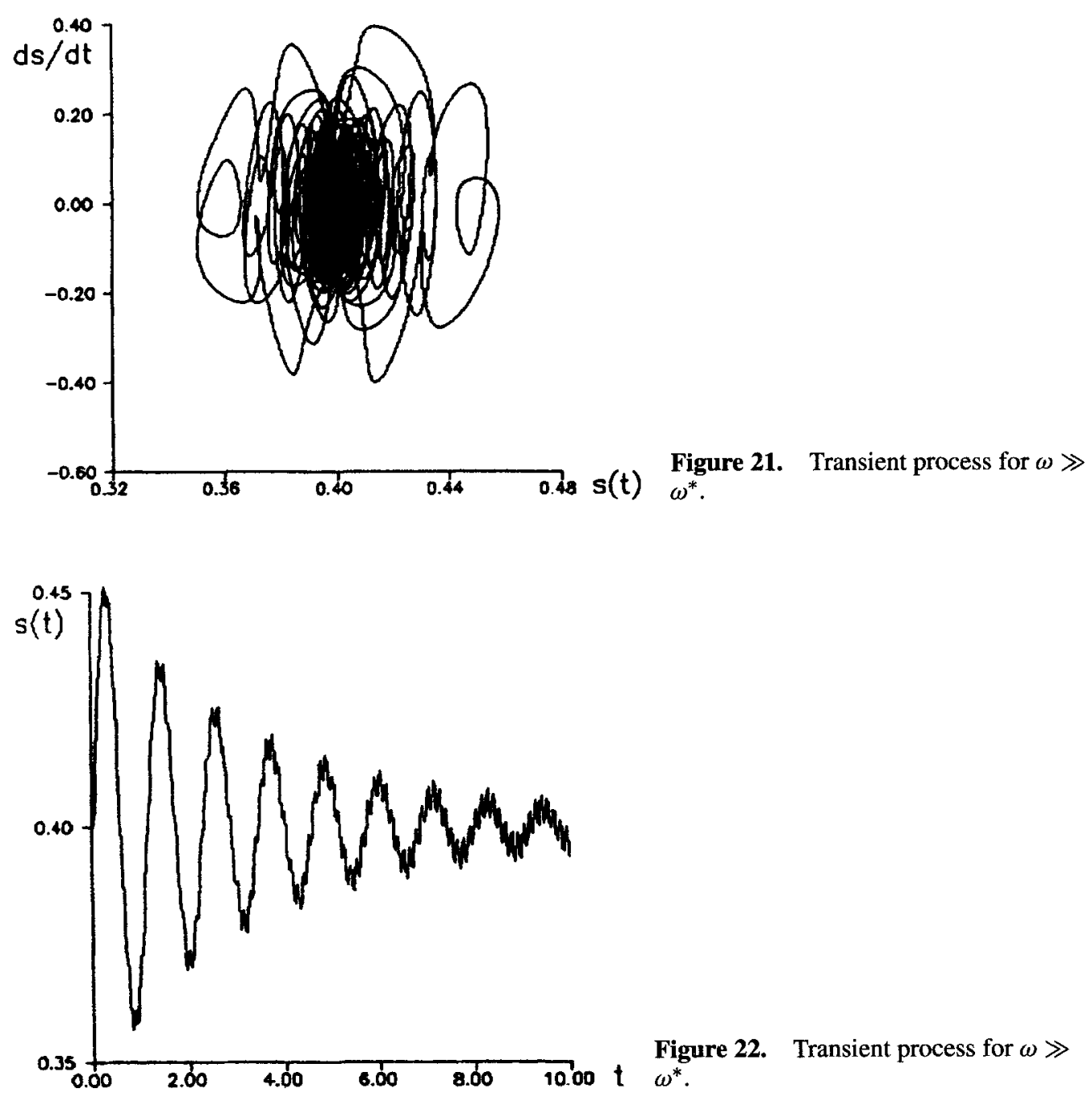

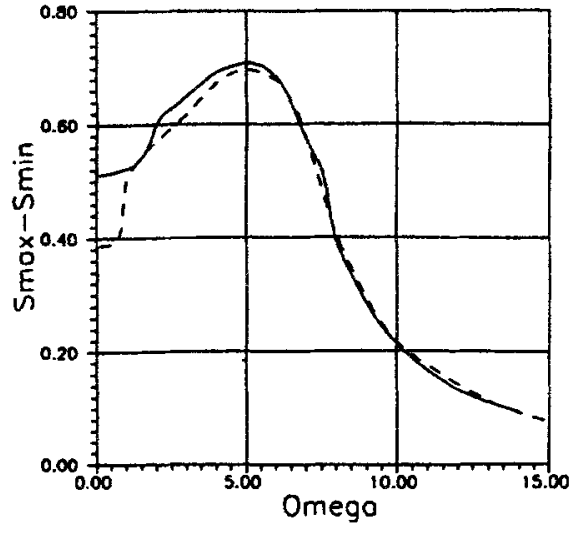

Figure 23. Comparison of resonance curves. 
Equation (66) is transformed into

$$
\frac{\partial \Gamma}{\partial \tau}=\frac{\partial^{2} \Gamma}{\partial r^{2}}-\frac{1+q(\tau)}{r} \frac{\partial \Gamma}{\partial r}, \quad r_{1} \leq r \leq 1
$$

with the boundary conditions:

$$
\Gamma(\tau)=1 ; \quad \frac{\partial \Gamma}{\partial r}=\frac{2 \Gamma}{r} \quad \text { at } \quad r=r_{1}
$$

The last one is the free surface condition.

Let us consider two cases: $\Omega \ll 1$ and $\Omega \gg 1$. For the first case

$$
\Gamma=\left[r^{q+2}+(q / 2) r_{1}^{q+2}\right] /\left[1+(q / 2) r_{1}^{q+2}\right] .
$$

If $q=0, \Gamma=r^{2}$ corresponding to solid body rotation. If $q \gg 1, \Gamma=r^{q+2}$, almost no rotation. If $q \ll-1, \Gamma=1-2 /|q|\left(r / r_{1}\right)^{q+2}$ and $\Gamma \approx 1$. For $\Omega \gg 1, \Gamma \equiv 1$. These results justify formulation of the problem using $\Gamma=$ const., which is correct for inviscid flow, for sufficiently high frequency, which is the most interesting case for the real LPE.

\section{Numerical analysis of VLPE}

We first want to estimate the engine parameters using an Otto-cycle. First, we estimate the natural oscillation frequency. Using (48), for typical values of the parameters, viz., $k=1.4, \sigma=0.25$, and $\Pi=4$, we have $\omega_{0}=6.26$ and $\omega=626 \mathrm{rad} / \mathrm{s}$. This value of $\omega$ corresponds to $n \approx 100 \mathrm{rev} / \mathrm{s} \approx 6000 \mathrm{rpm}$, which is typical for the maximum rpm of an internal combustion engine for automobiles. Thus, the vortex engine has good dynamic characteristics and can be started quickly.

The value of $\zeta$ is roughly estimated as follows. For the mean values we have $W=\Delta p Q_{v}$, or from (2) and (9)

$$
W=\zeta\left(\rho Q_{v}^{3}\right) /\left(8 \pi^{2} R^{2} h^{2}\right) .
$$

Let $W=100 \mathrm{~kW}$ ( $=134 \mathrm{hp}$ ), $R=0.1 \mathrm{~m}, h=0.1 \mathrm{~m}$, and $p=1000 \mathrm{~kg} / \mathrm{m}^{3}$. To estimate the value $Q_{v}$, we can use the formula $Q_{v}=\pi r^{2} h n$, where $r$ is the mean radius which can be found by setting $r_{1}=r_{2}$. Using (3) we find $r=R \sqrt{\sigma}$ and for $\sigma=0.25$, we have $r=0.5 R=0.05 \mathrm{~m}$. If $n=100 \mathrm{rps}, Q_{v}=0.078 \mathrm{~m}^{3} / \mathrm{s}$. From (67) then

$$
\zeta=\left(8 \pi^{2} R^{2} h^{2} W\right) /\left(\rho Q_{v}^{3}\right)=1664 .
$$

For the given dimensions and flow rate $Q_{v}$, we estimate the fluid velocity $V=5 \mathrm{~m} / \mathrm{s}$ at the entrance of the cylinder where $r=R$. This is a moderate velocity.

For numerical solution of (13), we use condition (20) and integrate for half a period until $\dot{y}=0$. At this moment, if (22) is satisfied, we have $y_{0}$ and all other engine characteristics. Otherwise, we use secant or Newton's method to find a new $y_{0}$ and start again until (22) is satisfied.

For the example considered (i.e. $W=100 \mathrm{~kW}=134 \mathrm{hp}, R=h=0.1 \mathrm{~m}, \zeta=$ $5000, V=5 \mathrm{~m} / \mathrm{s}$, and $\sigma=0.4$ ), we obtained numerically $\epsilon \approx 10, \eta=60 \%$, and a large margin for stability, $S_{t}=72$, corresponding to the acceleration $a_{c} \approx 1300 \mathrm{~g}$ at the maximum compression point. In order to have an acceptable efficiency value, we assign 


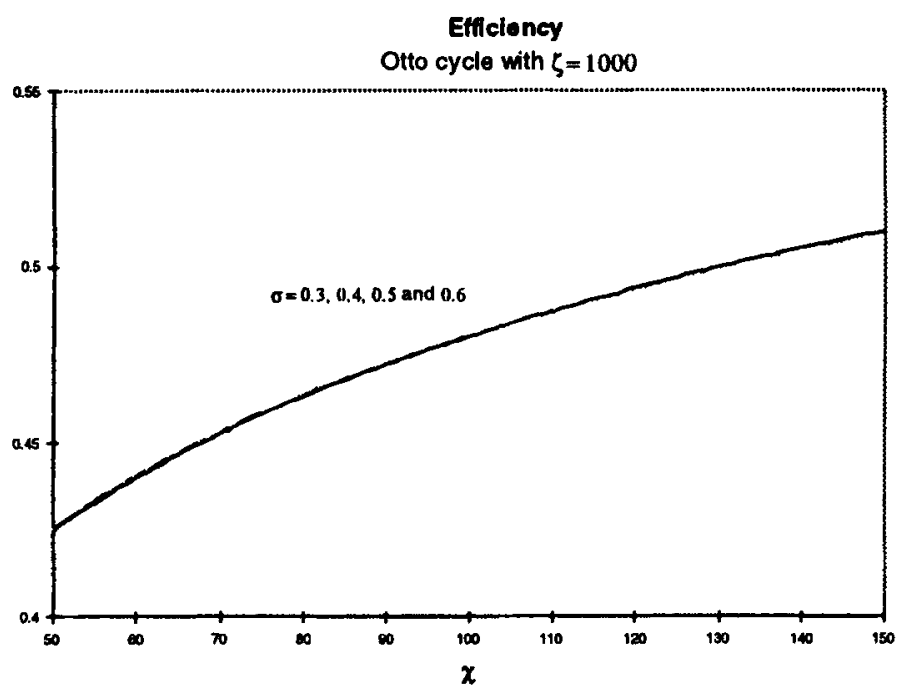

Figure 24. Efficiency for Otto cycle with fixed $\zeta$.

the compression ratio, (19). We want to have a maximum magnitude of oscillation, in which case the maximum radius $r_{1}=R$. This yields $y_{0}=1-\sigma$, which together with (19) gives $\sigma=0.55$ and $y_{0}=0.45$.

We chose the following parameters for the experimental device: $R=0.1 \mathrm{~m}, b=$ $0.019 \mathrm{~m}, h=0.3 \mathrm{~m}$ and $v_{\varphi}=5 \mathrm{~m} / \mathrm{s}$. From numerical calculations, $|\dot{y}|_{\max }=0.03$. The corresponding working parameters are: $\beta=4.49, \zeta=20000, f=1.05 \mathrm{~Hz}, a_{c}=806 \mathrm{~g}$, $S_{t}=1986, W=669 \mathrm{~kW}, \Delta p=1.12 \mathrm{~atm}, Q_{\max }=0.028 \mathrm{~m}^{3} / \mathrm{s}, p_{R 1}-p_{R 2}=2.25 \mathrm{~atm}$, $p_{R 1}=2.25 \mathrm{~atm}$, and $p_{R 1}-p_{r 1}=1.13 \mathrm{~atm}$. The low frequency here is only for detailed investigation of the process and is not typical for the vortex engine. All these parameters are used and verified in our experiments. A distinct feature of the vortex liquid piston engine

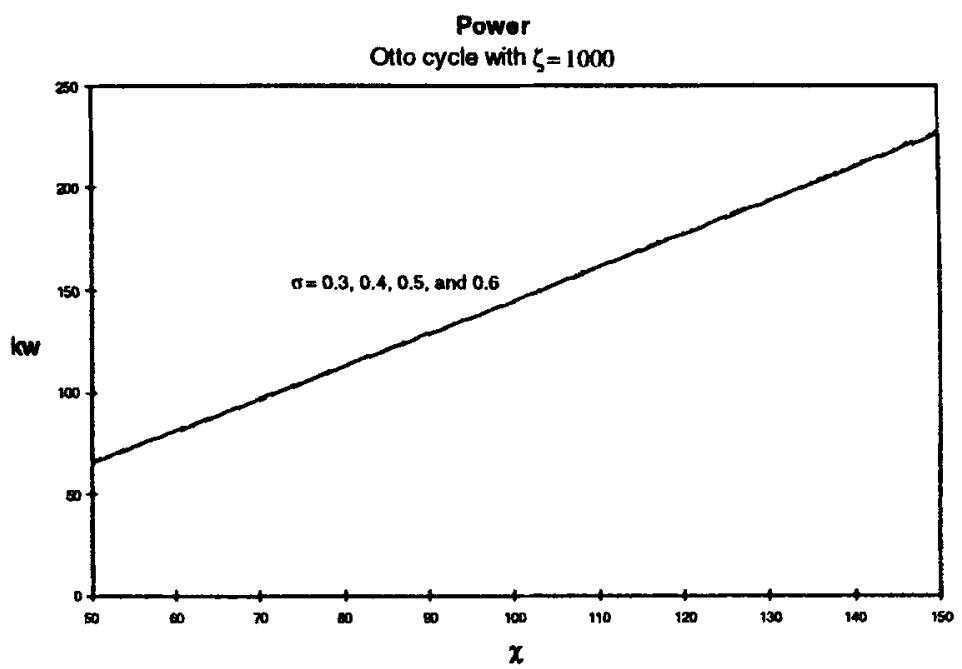

Figure 25. Power for Otto cycle with fixed $\zeta$. 
Frequency

Otto cycle with $\zeta=1000$

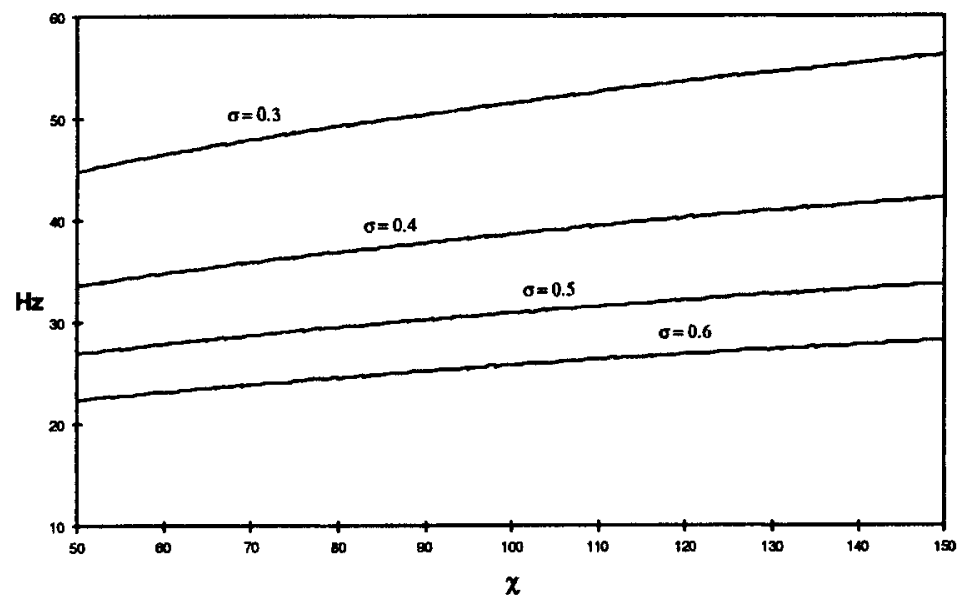

Figure 26. Frequency for Otto cycle with fixed $\zeta$.

is that its efficiency increases with increasing power. This can be explained by the increase of the oscillation amplitude of the radii of the cavities and therefore of the compression ratio $\epsilon$. The vortex engine differs significantly from the conventional internal combustion engine where $\epsilon$ is fixed and $\eta$ decreases with increasing power.

To complete the numerical analysis, we calculated the power, efficiency, and frequency of this engine for Otto cycle. Figures 24-26 show the results for a constant $\zeta=1000$. From these figures, we can see for the Otto cycle:

(1) both engine power and efficiency increase with $\chi$, but change little with $\sigma$.

(2) engine frequency decreases with $\sigma$ (figure 26). This is a unique feature because it shows that the frequency increases with the mass of the oscillating system.

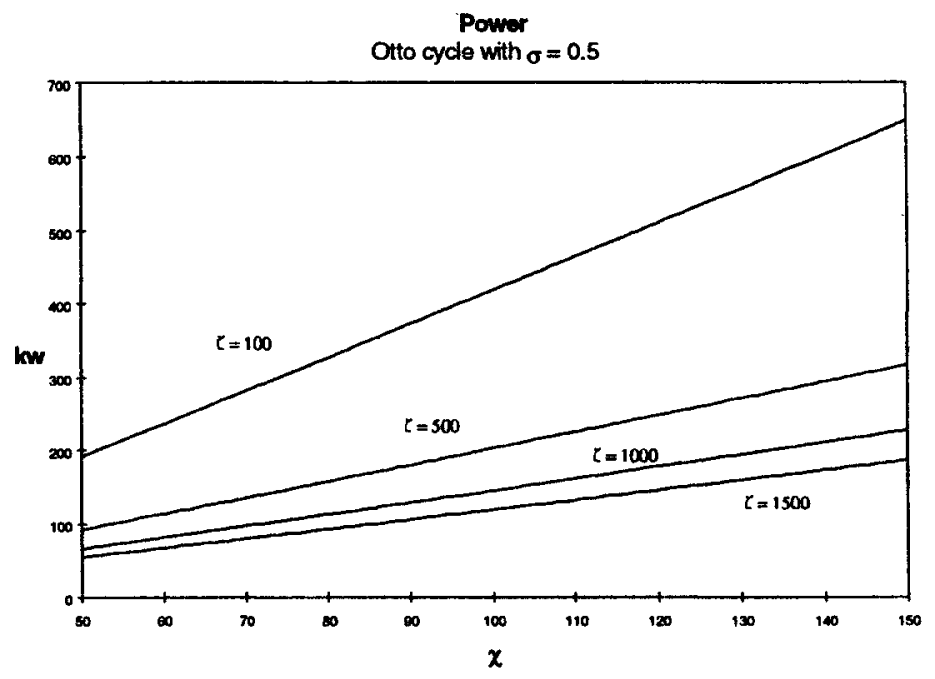

Figure 27. Power for Otto cycle with fixed $\sigma$. 


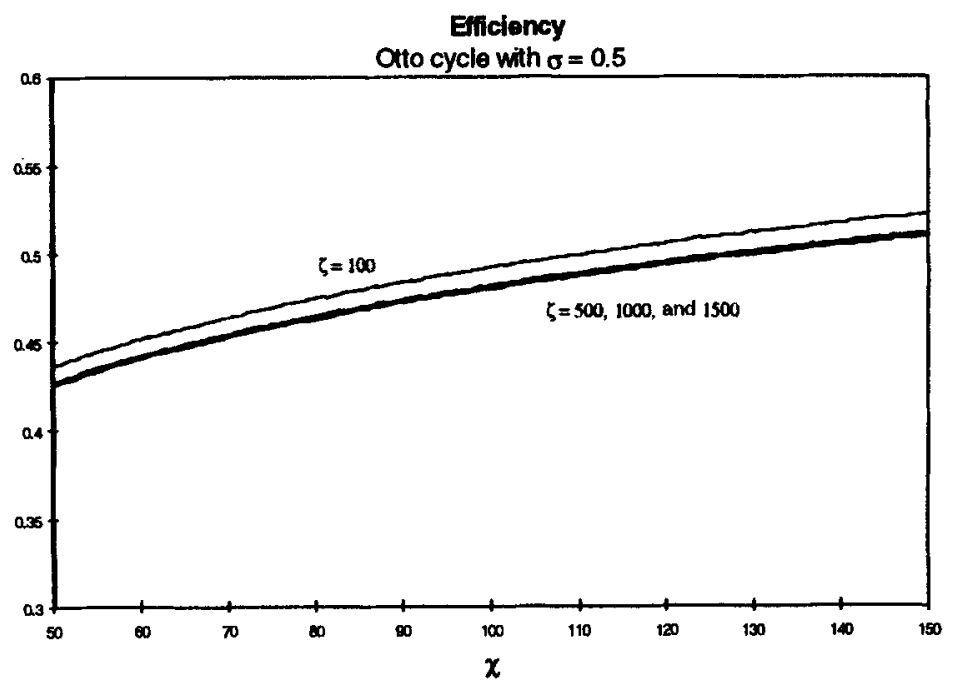

Figure 28. Efficiency for Otto cycle with fixed $\sigma$.

Figures 27-29 show the results with a constant $\sigma=0.5$. From these figures, we can see for the Otto cycle that power, efficiency, and frequency, all decrease with increasing $\zeta$.

Figures 30 and 31 show the case when $\chi$ is small. From these we can see for the semi-Otto cycle:

(1) engine power increases with increasing $\sigma$ (figure 30b) and $\chi$ (figure 30a);

(2) engine power has a maximum with respect to $\zeta\left(\zeta_{\max } w\right)$ (figures $30 \mathrm{a}$ and b); with increase of $\sigma$ (less fluid), $\zeta_{\max w}$ decreases (figure 30b); however, if $\chi$ is large enough, $\zeta_{\max w}$ is almost constant with increasing $\chi$;

\section{Frequency}

Oto cycle with $\sigma=0.5$

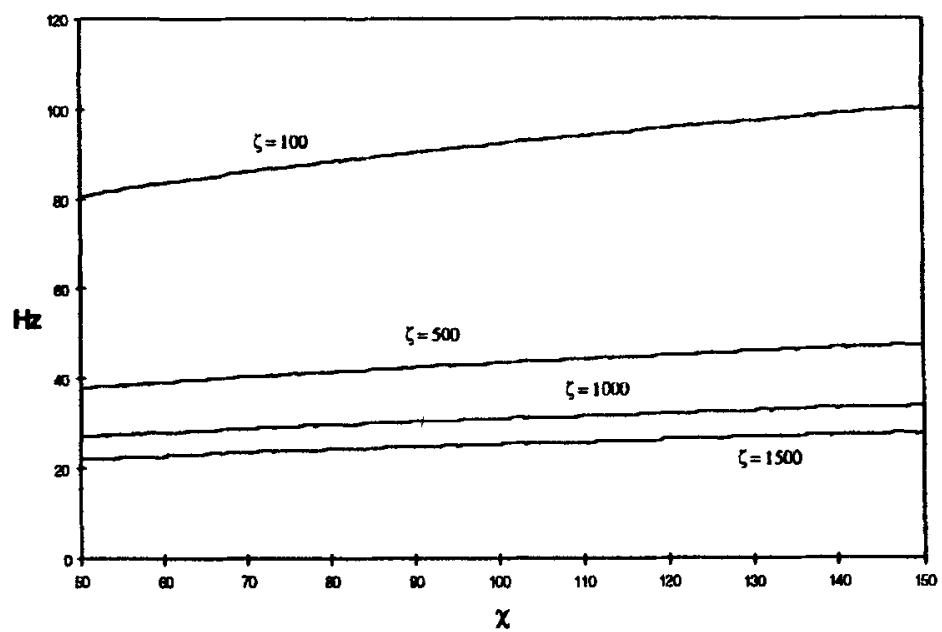

Figure 29. Frequency for Otto cycle with fixed $\sigma$. 
Power as a function of $\zeta$ and $\chi$

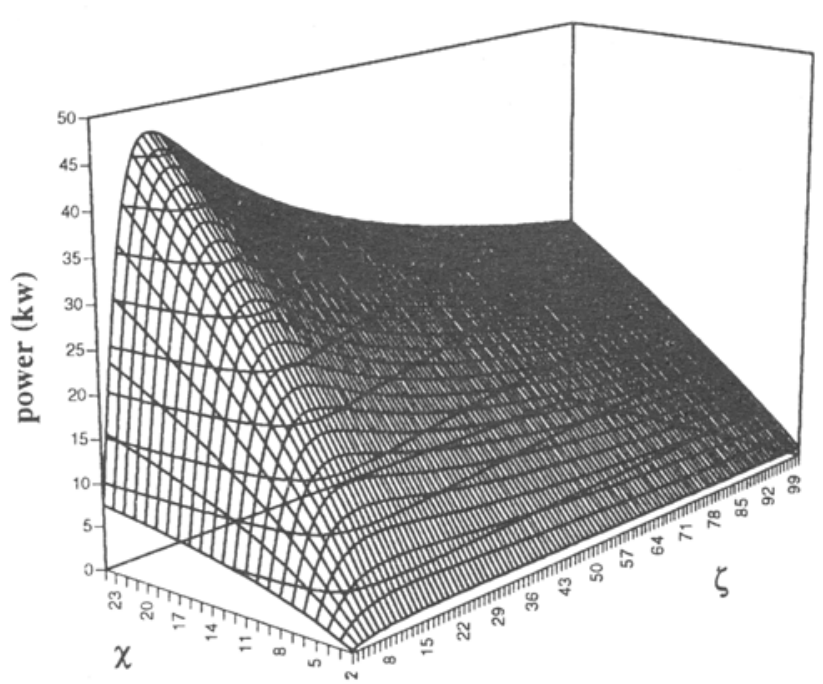

a)

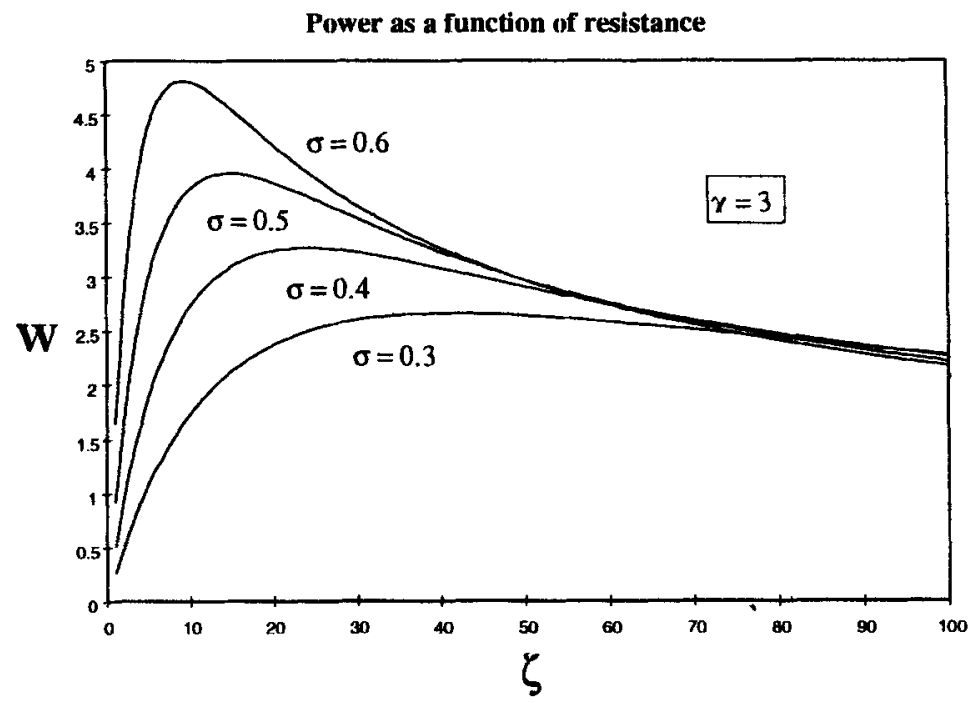

b)

Figure 30. Power for the semi-Otto cycle. 


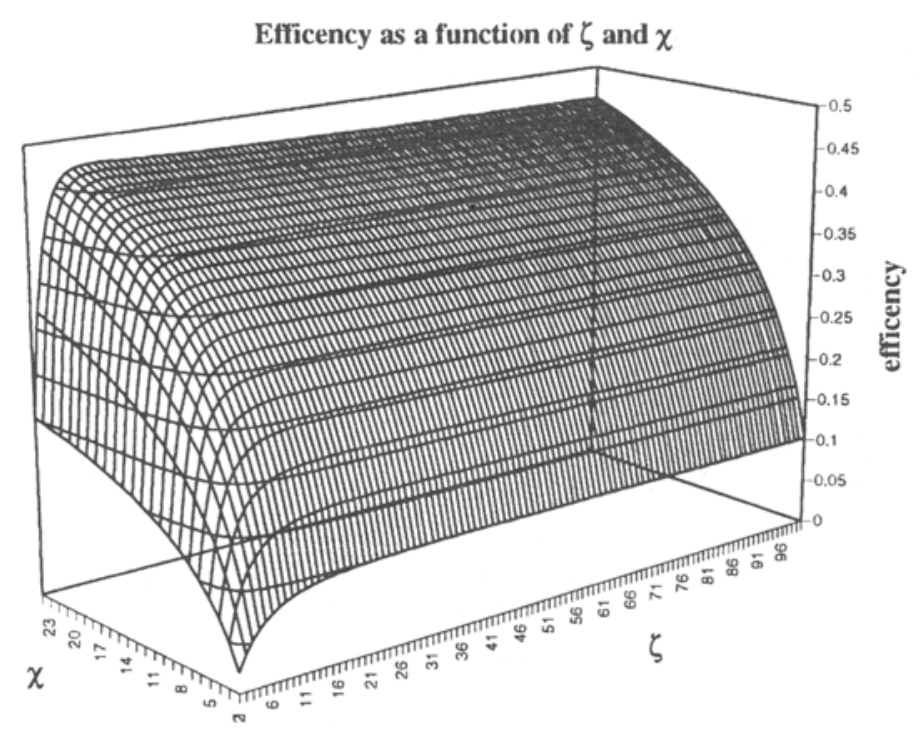

a)

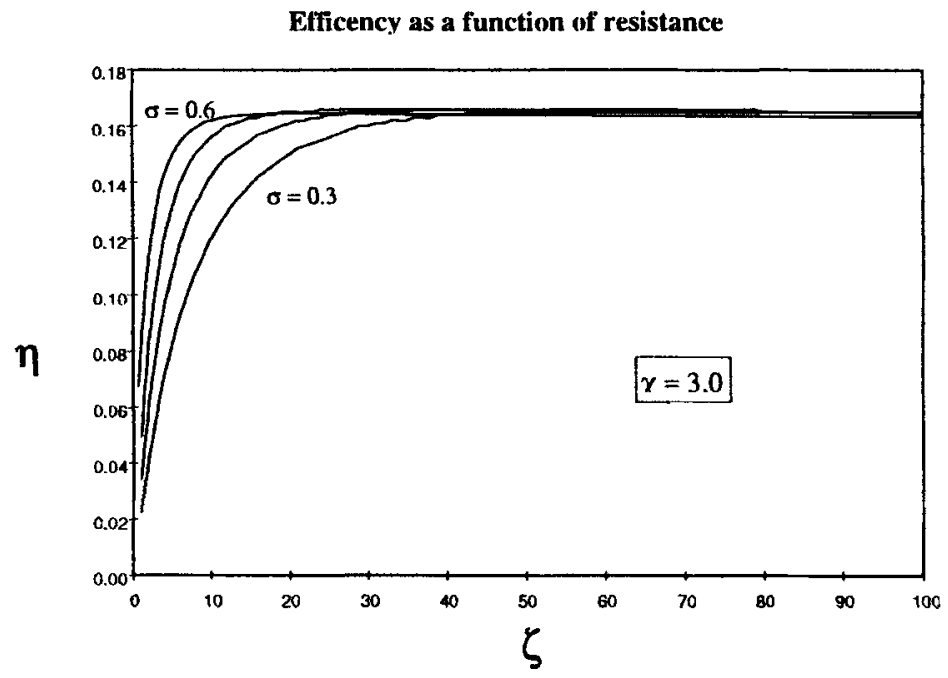

b)

Figure 31. Efficiency for the semi-Otto cycle. 


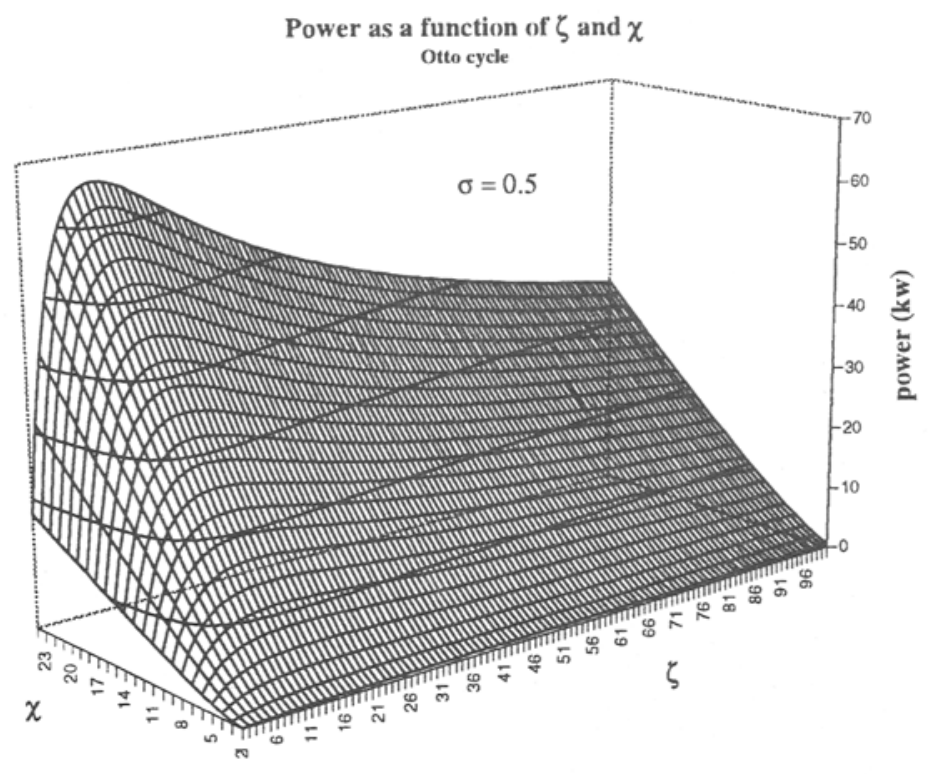

a)

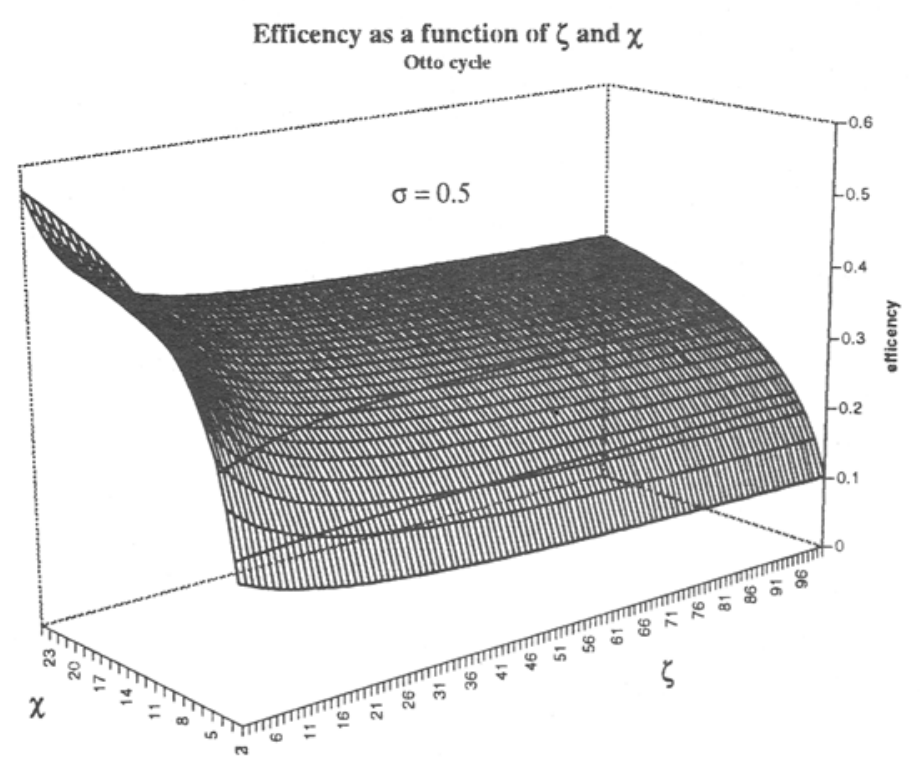

b)

Figure 32. Power and efficiency for Otto cycle. 
(3) engine efficiency increases with increasing $\sigma$ (figure 31b) and $\chi$ (figure 31a);

(4) engine efficiency has a maximum with respect to $\zeta\left(\zeta_{\max _{\eta}}\right)$ (figure 31a); with increase of $\sigma$ (less fluid), $\zeta_{\max _{\eta}}$ increases (figure 31b); however, when $\zeta$ is large enough, engine efficiency will be almost constant (figure 31a).

Figure 32 shows the similar case for the Otto cycle.

\section{Engine design and experimental analysis}

After finding all the necessary relationships and the optimized design parameters, we built a working model using water and air. Figure 33 shows our first design. It consists of two cylinders connected by two tangential channels and a check valve, so that water can flow in each channel only in one direction.

We used an external oscillating circuit to control all four solenoid valves (one air inlet and one exhaust valve for each of the chambers). The control circuit is shown in figure 34, where $R 2$ can be adjusted to achieve different oscillation frequencies.

After the first prototype was built and tested, we discovered a serious problem with this design. We had assumed that high-speed water would enter the chamber and rotate inside it to create a stable axisymmetric cavity. However, a very complicated turbulent flow without swirl and cavity was produced (figure 35) because of the tangential outlet whose lip generates a big disturbance due to flow impingement and thus an obstacle to the rotating flow. A swirling wall jet of water hits the outlet conduit and is deflected from this obstacle back to the centre of the chamber.

In order to eliminate this "outlet effect" and provide a smooth surface for the rotating flow, we completely blocked the upper channel (figure 36a) and divided the lower one into two halves (figure 36b): one half is for incoming flow and the other half for outflow. This time swirling flow and a cavity were created. However, on injection of pressurized air into the cavity, the cavity immediately collapsed. After careful analysis, we found the main

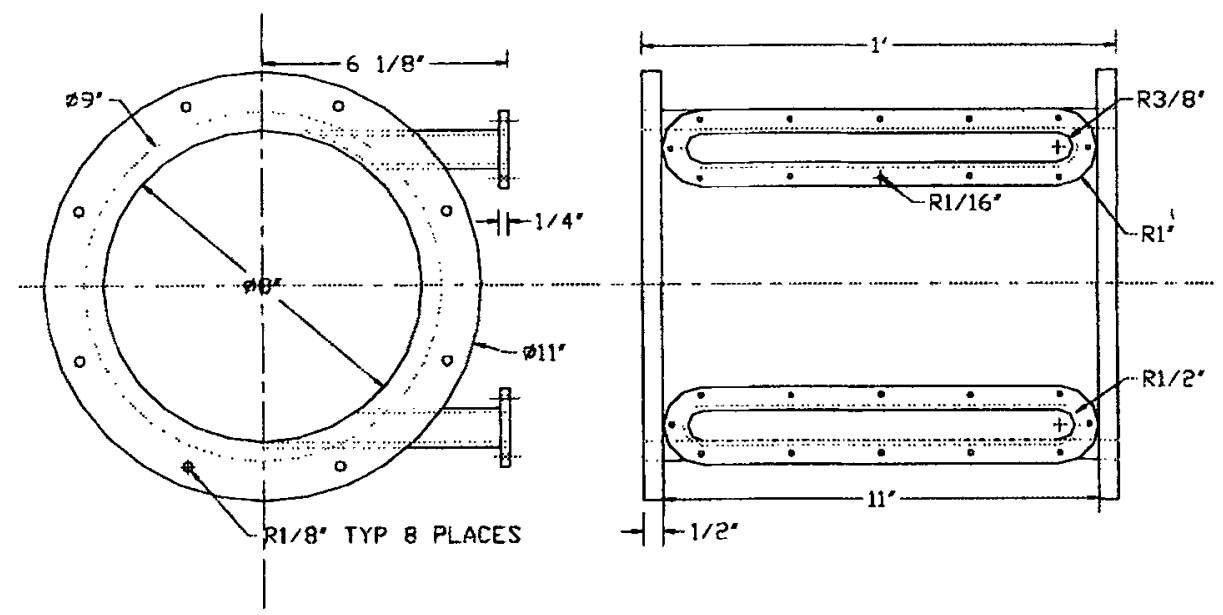

Figure 33. Our first design. 


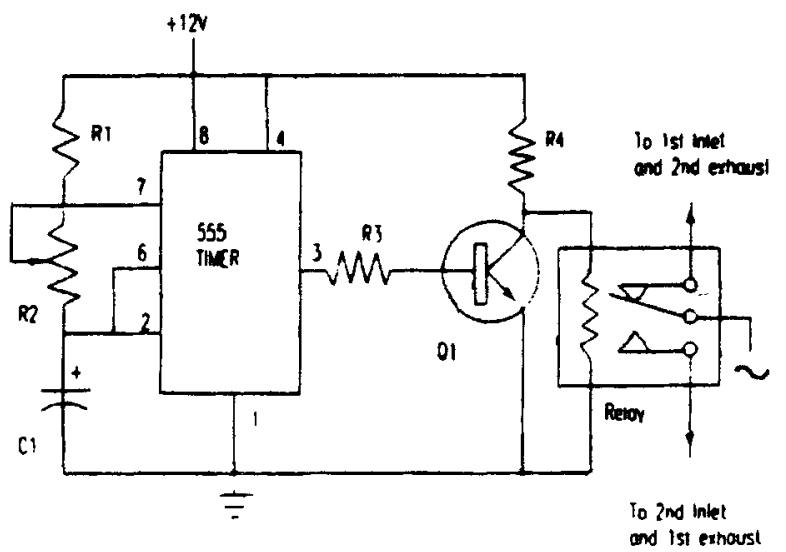

Figure 34. Control circuit.

reason is because the outlet is not axisymmetric. This breaking of the system symmetry causes the cavity to be destroyed.

Since axisymmetry is crucial, it was thus necessary to modify our model. Figure 37 shows a meridional cut of the actual modification where the block in the middle prevents air from exiting with the liquid when this model works horizontally.

Figure 38 shows a picture of the experimental configuration. As expected, the cavity created with this modified model expands smoothly when pressure is applied. Therefore, we have achieved a working chamber with an expandable and contractible cavity inside it. The cavity created is quite stable as predicted by our theory. All the system parameters measured, such as oscillation frequency, flow rate, and power, are comparable to our theoretical model and numerical results. Figure 39 shows the minimum cavity in the periodic working engine. One can see from these pictures that the cavity, though successfully generated, is not quite axisymmetric. The reason is the non-axisymmetry of the inlets and outlets of the system and machining roughness. This is why further redesign was necessary.

We realized that the most important design goal is to make the system as axisymmetric as possible, because any non-axisymmetry will most likely cause the cavity to be unstable or to collapse under sudden application of pressure. In order to have an axisymmetric inlet, we decided to use a distributor as shown below (figure 40). This distribution forms evenly distributed water jets and helps us create a more stable cavity.

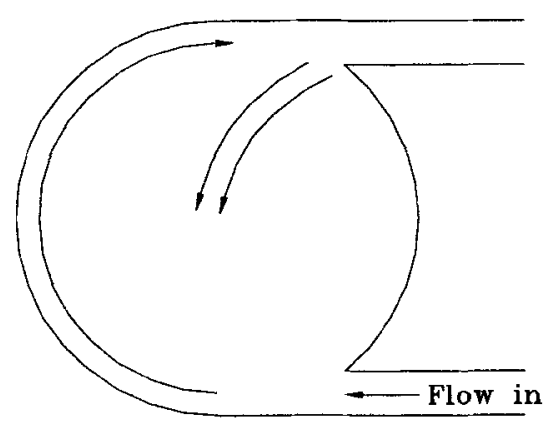

Figure 35. Cavity did not form in the first model. 


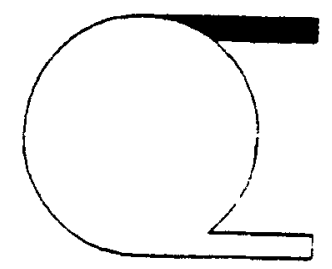

(a)

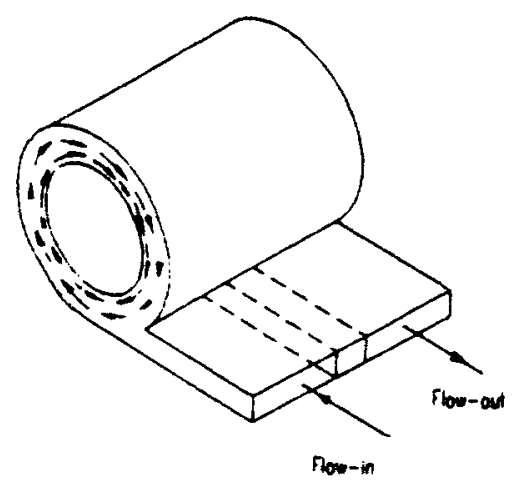

(b)

Figure 36. Blocked upper channel and divided lower one.

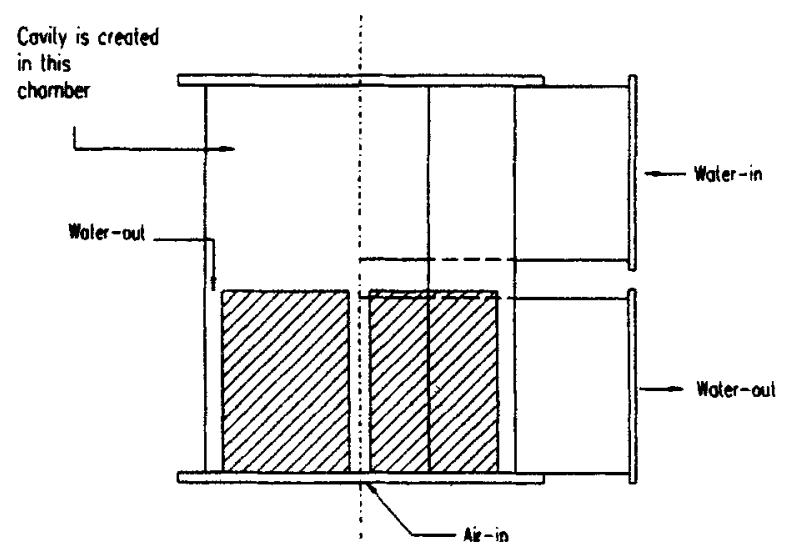

Figure 37. Our modified model.

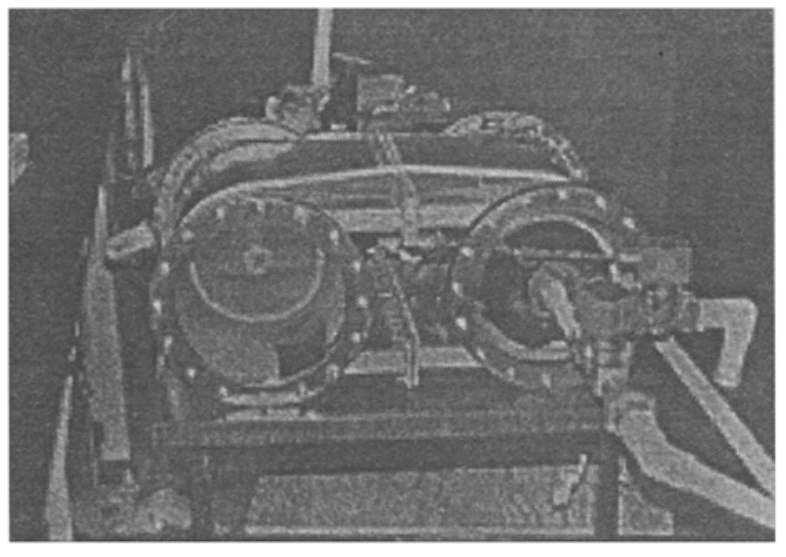

Figure 38. Experimental configuration. 


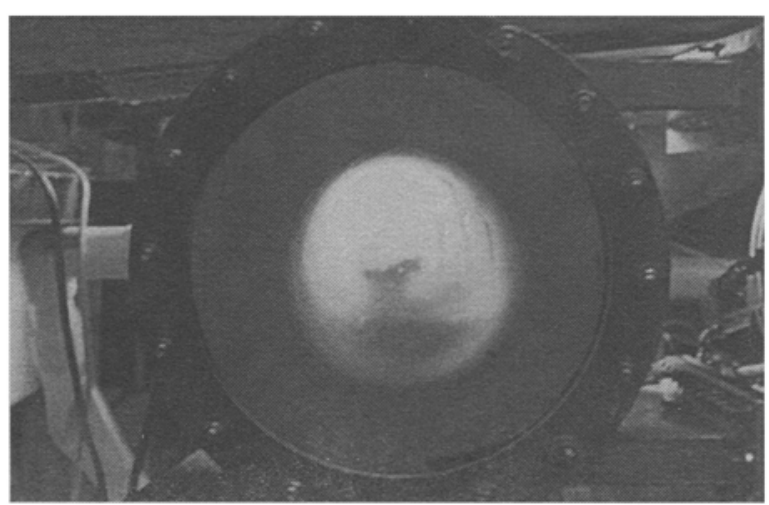

Figure 39. Minimum cavity of the periodic working engine.

For simplicity of pipe connections, we used a horizontal configuration for the first experiment, which caused an unstable cavity and air-water mixing. Therefore, the next design used a vertical configuration.

\section{Engine redesign and experimental analysis}

In order to avoid air/water mixing which occurred in the first model, we decided to adopt a vertical configuration with swirlers (figure 41 ). From the previous model, we realized that the most important design goal is to make the system as axisymmetric as possible, because any non-axisymmetry will most likely cause the cavity to become unstable or to collapse on the sudden application of pressure.

In order to have an axisymmetric inlet, we designed swirlers as shown in figure 42 . This swirler forms evenly distributed water jets which helps to create a more stable cavity.

The number of blades on the swirler is not arbitrary and is related to the dynamics of the system. Its relationship with hydraulic load $\zeta$ and frequency $f$ is shown in figures 43 and 44 respectively.

If we want this model to work at a modest frequency, say $5 \mathrm{~Hz}$, just to demonstrate the principles, from figure 44 we find the corresponding $n$ is 12 .

The geometry of the swirler is also related to the dynamics of the system and has been carefully designed. Figure 45 shows a typical entrance geometry where angle BAD forms a

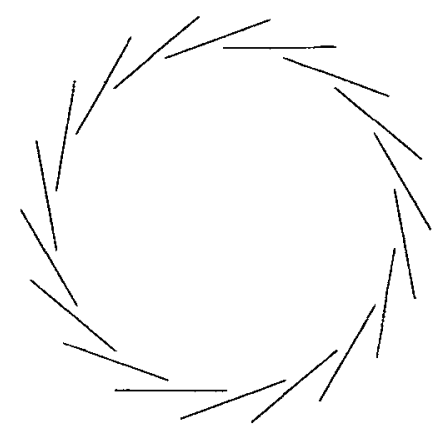

Figure 40. Water distributor. 


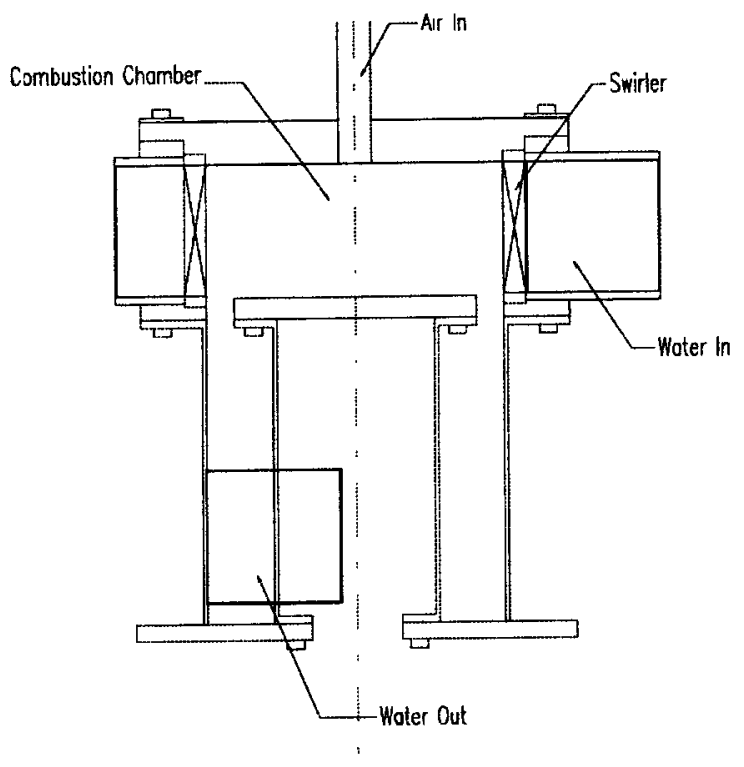

Figure 41. Vertical configuration.

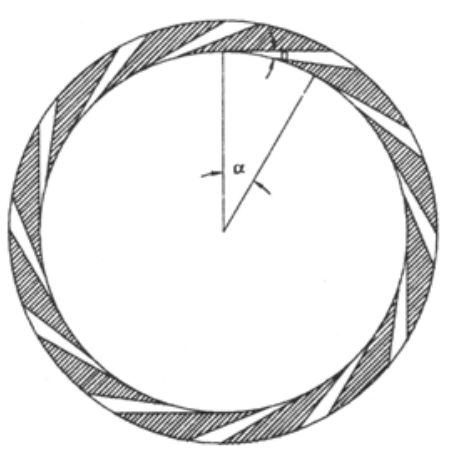

Figure 42. Swirler.

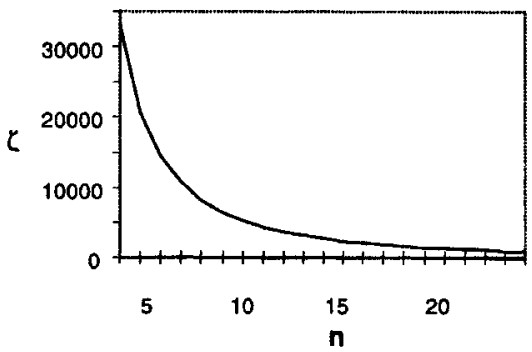

Figure 43. Load coefficient $\zeta$ as a function of $n$.

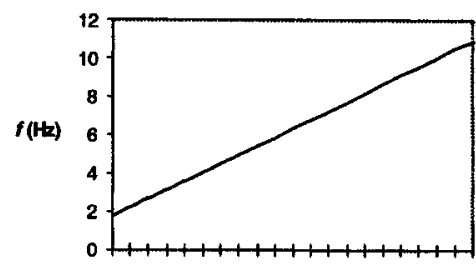
5
$10 \quad 15 \quad 20$
Figure 44. Oscillating frequency as a function of $n$. 


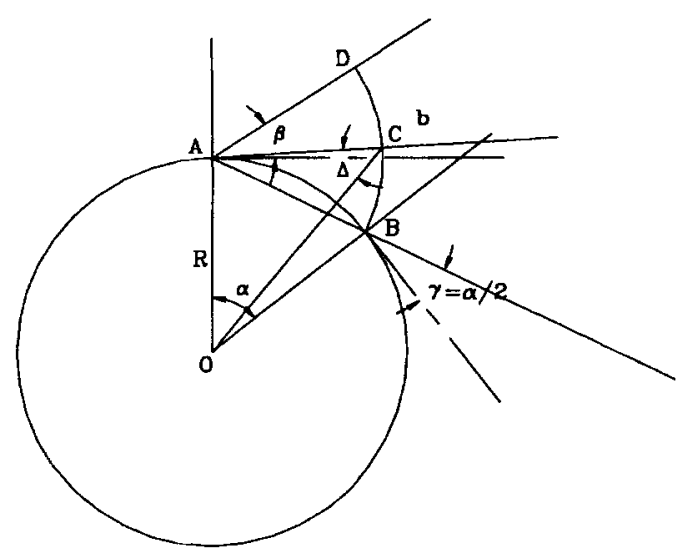

Figure 45. Swirler geometry.

single entrance. It has been proven that when the two blades intersect at point $\mathrm{A}$, circulation is maximum for a given flow rate. As shown before, we introduced a new parameter $\theta=v_{r} / v_{\varphi}=|\dot{y}|$. From figure 45 , it can be found that $\theta=\cot \Delta=\left(1-\sin ^{2} \Delta\right)^{1 / 2} / \sin \Delta$. When $\beta=0$ (tangential inlet), we have

$$
\theta=\left(4 \sin ^{2} \frac{\alpha}{2}+1-4 \sin \frac{\alpha}{2} \sin \frac{\alpha}{4}-\cos ^{2} \frac{\alpha}{4}\right)^{1 / 2} / \cos \frac{\alpha}{4}
$$

where $\alpha=2 \pi / n$.

We also improved the control system. First, we removed the external oscillator and used the signals from engine check valves as control parameters. Then we replaced the mechanical relay control system with a new microcontroller and solid-state relays. In order to achieve the semi-Otto cycle, air supply valves $A_{1}$ and $A_{2}$ (figure 46) must be open for only a very short time ' $t$ ' (to mimic combustion), and this time should be adjustable. $\mathrm{A}_{i}, \mathrm{E}_{i}$, and $\mathrm{I}_{i}$ are the air supply, exhaust and inlet check valve for chamber $i$ respectively.

Figure 47 shows the control sequence for the valves having a very short adjustable time $t$ for the air inlet valves to simulate combustion.

We use a Basicon MC-1Z microcontroller to implement this control sequence. It is a fully self-contained general purpose programmable controller with CPU, RAM, ROM, real-time-clock, I/O, communications circuitry and a resident BASIC language.

Figure 48 shows the setup of the new model and figure 49 is a close-up to reveal the cavity.

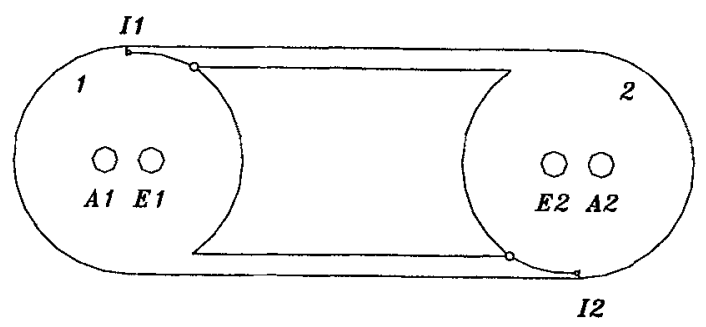

Figure 46. Schematic of the engine control. 


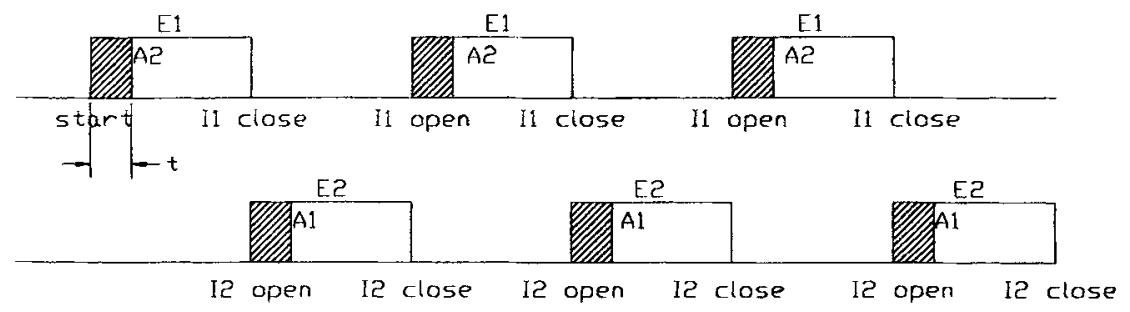

Figure 47. Timing diagram.

\section{Summary}

Here we briefly review the basic principles and potential advantages of the VLPE. The two cylinders are interconnected by two tangential, unidirectional flow channels containing hydromotors. Because of the tangential inlets, the liquid is caused to rotate in each cylinder and creates a vortical liquid body with a cylindrical cavity in the centre. Each cylinder has an inlet valve for the intake of fuel and air, as well as an associated exhaust valve. The cylinders can either have an electric spark plug or work in a diesel mode with fuel injectors.

The rotating liquid in the engine simultaneously performs three functions: (a) it creates a stable cavity; (b) it stores rotating kinetic energy (a liquid "flywheel"); and (c) it has effective elastic properties for damping detonation and permits a higher compression ratio than that of the conventional engines.

Our mathematical model describes the behaviour of this two-cylinder engine. Here we present the design and operating parameters of a $100 \mathrm{~kW}$ engine. The diameter of each cylinder is $0.1 \mathrm{~m}$, the cross-sectional area of the tangential inlets and outlets is $20 \mathrm{~cm}^{2}$ and the velocity of the input liquid is $10 \mathrm{~m} / \mathrm{s}$. The corresponding pressure drop across a hydromotor is $14 \mathrm{~atm}$. The thermal efficiency for the Otto cycle is 0.6 and the compression ratio is about 10 . The stability criterion for this example is 72 , which ensures a large margin of stability. The acceleration in the maximum compression stage is very large

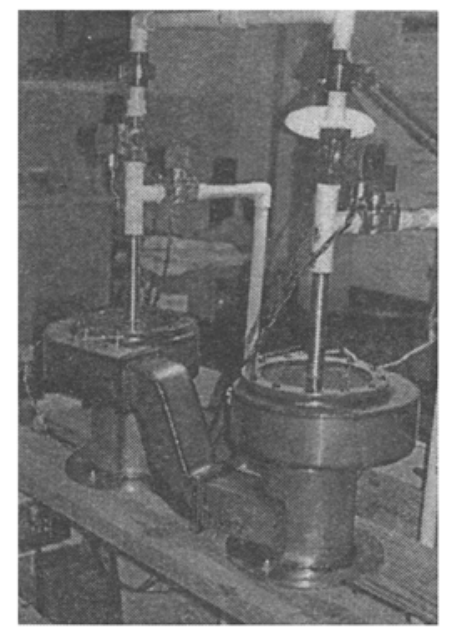

Figure 48. Setup of the engine. 


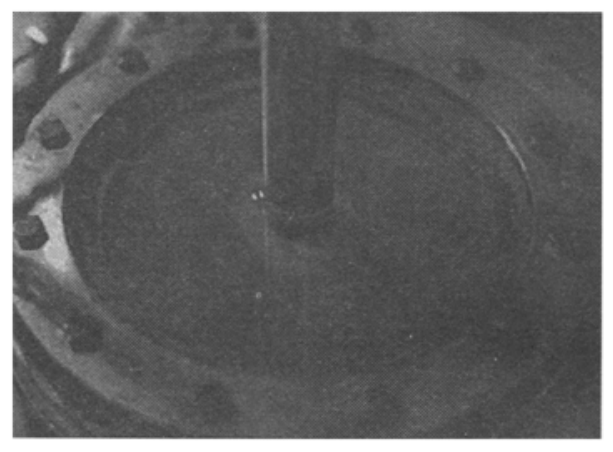

Figure 49. Cavity.

- approximately $1300 \mathrm{~g}$. When running in diesel mode, the power and efficiency of an engine of the same size will increase significantly because of higher compression ratio. The preliminary experiments have demonstrated the stable cavity in the "cold" model of vortex liquid piston engine.

The engine employing a rotating liquid as a piston has the following advantages:

(1) It is small, lightweight and generates large amounts of power;

(2) It is simple to service and repair, having low maintenance;

(3) The damping of detonation allows high compression ratios, and thus significantly improving thermal efficiency; emission of environmentally damaging pollutants is dramatically reduced;

(4) If a receiver is available to act as an accumulator, a new type of hydrodynamic braking is possible; part of the kinetic energy might be transferred to the receiver by the hydromotor acting as a hydropump to provide energy to the receiver.

\section{Other vortex machines - concepts and applications}

We are using the features of vortex flows outlined in the introduction to develop several vortex machines, briefly mentioned below.

Vortex thruster (VT): It is essentially a bladeless helicopter, a real 'flying saucer' of practical relevance. The basic working principle of this device is to create a strong swirling flow which produces a low pressure above a surface, thus generating lift or thrust. The swirl in the incoming flow is achieved via an open vortex chamber, and the resulting low pressure rarefaction is intensified by an airfoil-shaped diffuser, which ensures an attached flow without separation. Referring to figure 50, the propulsion device has an open vortex chamber (5) which serves to create a strong swirling open fluid jet; (1) is a source of pressurized fluid, and (2) is a header for uniform flow distribution. The flow enters the swirler (3), acquires strong angular momentum and exits over the diffuser (4), where it reverses direction (by the Coanda effect) and is ejected into the ambient fluid. Such a flow is like an artificial tornado, creating a strong rarefaction (low-pressure) zone on the upward-facing, internal top-surface of the vortex chamber. Since there is a relatively higher 


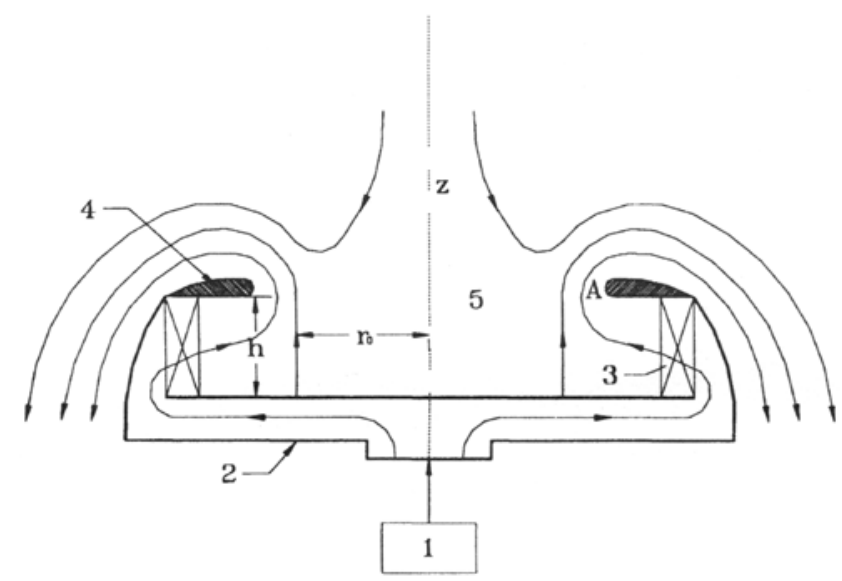

Figure 50. Vortex thruster.

pressure on the external bottom-surface of the chamber, a lift force is generated by this pressure difference.

Additional rarefaction is created by the flow over the upper surface of the diffuser by its airfoil shape. Operating parameters for the vortex chamber must be determined to avoid separation over the diffuser near point A. Such attached flow over the diffuser is possible since the Coanda effect is much stronger in the presence of swirl and is also stronger in turbulent flow. Physically, the difference between conventional jet propulsion and our vortex thruster is that, in the former, the net force generated and the power requirements are determined by the same axial velocity component. In our vortex thruster, a large net force is generated primarily by the tangential velocity. component, with only a relatively small axial flow rate; hence, relatively small power is required. Preliminary experiments using a simple device to model a thruster have verified that thrust is, in fact, generated in the same direction as the exit flow. Our initial conservative estimates indicate that a thruster with a $1 \mathrm{~m}^{2}$ chamber area can generate 4 tons of thrust requiring 17 times less energy than a conventional jet. Thus, this vortex thruster, which can operate in air or water, has significant potential applications in enhancing or replacing conventional thrust/lift devices, e.g. propellers and wings on helicopters, aircraft, and ships; in particular, a bladeless helicopter will be a highly desirable innovation from the viewpoint of maneuverability and safety.

Vortex suction device (VSD): Based on essentially the same principle as the vortex thruster, this device utilizes the ability of swirling jets (zone $C$ ) to create a strong reverse flow near the axis (figure 51, zone A). The velocity in the zone $\mathrm{A}$ is inversely proportional to the distance $R$ instead of $R^{2}$ for an ordinary vacuum device. The swirling flow (in this directionally focused suction device) imparts a wide range of strong suction by drastically reducing the energy consumption, in a manner analogous to that discussed for the thruster. 


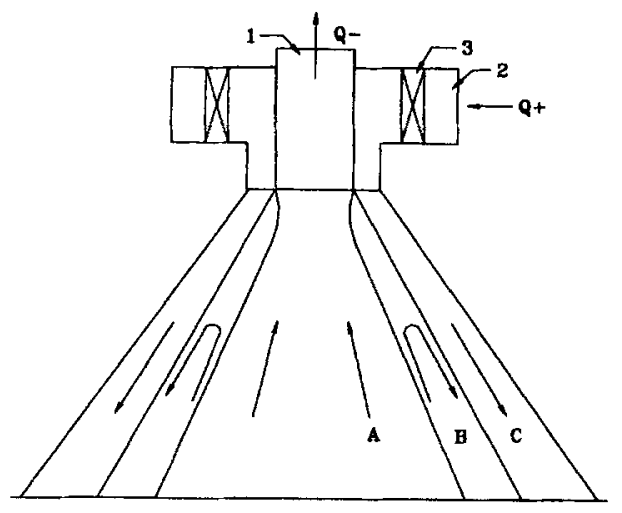

Figure 51. Vortex suction device.

Vortex chemical reactor (VCR): In the condensed stable state (see the introduction) of a solid-gas or solid-liquid mixture in a vortex chamber (figure 2), a densely packed, homogeneous fluidized layer is formed near the periphery of the cylindrical chamber, which enhances heat and mass transfer; this stable state, having a high interfacial surface area, is independent of the flow rate over a wide range. These properties of homogeneity and quasicrystalline structure provide high levels of heat and mass transfer, shown schematically in figure 52. The layer (3) is held in a rotating liquid or gas which is used as the heat transfer medium. The fluid is introduced tangentially through the cylindrical periphery of the chamber and exits through a central diaphragm in one or both end faces. In figure 52, the fluid enters through an inlet (1) into the device (5), typically a single- or multi-threaded

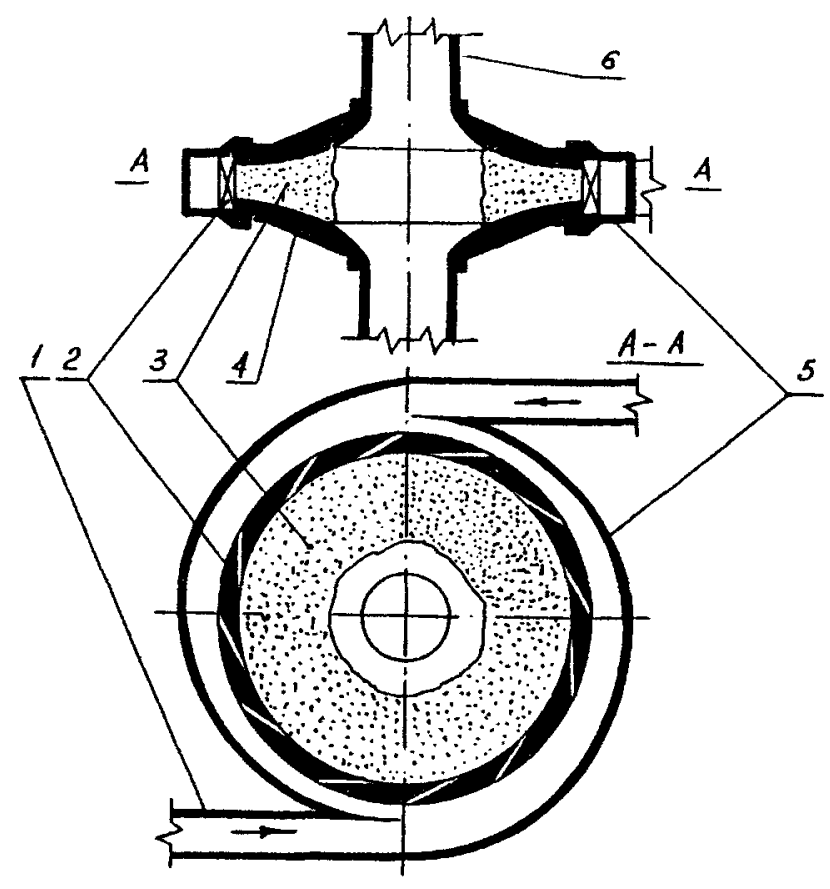

Figure 52. Schematic of VCR. 


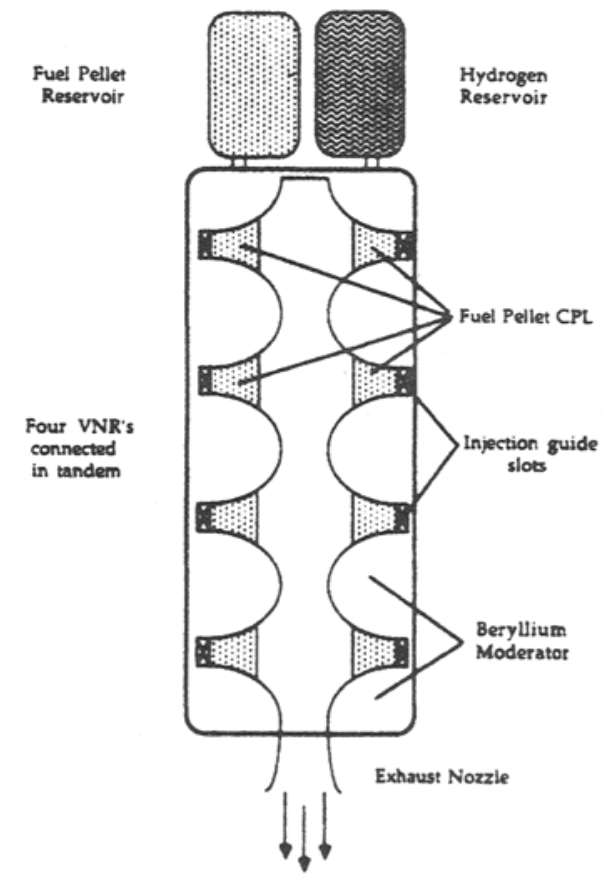

Figure 53. Schematic of VNR.

scroll. This allows uniform distribution of the fluid along the guide device (2). The fluid then enters the vortex chamber with appropriately profiled walls (4) and leaves through exits (6). The fluid plays a two-fold role: First, it removes heat as it flows through the particle layer; second, rotation of the fluid ensures confinement of the layer.

The high centrifugal acceleration makes the VCR a more efficient fluidized bed than conventional technology which utilizes gravity. VCR is particularly well-suited in chemical reactors using catalysts, and in burning pure, granular aluminum as fuel for military and aerospace applications. The swirling flow in the vortex chamber with the exhaust at the centre guarantees residence of the particles (catalysts, reacting fuel, etc.) within the chamber while they are essentially consumed, thus significantly increasing efficiency and reducing wastage. The fluidized layer would also be useful in the agriculture industry for efficient surface drying and separation of grain.

A specific potential application of this technology is a vortex nuclear reactor (VNR), which employs a concentrated pseudofluidized layer (CPL). The CPL consists of small spherical fuel pellets rotating in a special vortex chamber surrounded by a fluid (liquid or gas) acting as a heat transfer medium. To illustrate its basic operation, the active section of the VNR for space applications is shown schematically in figure 53. It consists of granular fissionable material formed into a CPL in a special vortex chamber.

The VNR consists of several parts: fuel pellet and hydrogen reservoirs, fuel pellet CPL, injection guide slots, beryllium moderator, and exhaust nozzle. It has the following possible uses:

(i) nuclear reactor with high specific power output utilizing radically reduced amounts of nuclear fuel in continuous operation; 
(ii) a nuclear reactor engine; and

(iii) high neutron flux research reactor and breeder.

The VNR has significant safety advantages over conventional nuclear reactors because of minimal amount of fuel pellets in residence in the chamber at any time, very high specific power, compact size for ease of maneuverability, and a self-cleaning capability for encrusted fuel elements. This latter feature permits use of the VNR with heat transfer media such as oil or sea water.

Bubbling centrifuge $(B C)$ : Vortex bubbling devices are used to enhance heat and mass transfer processes between liquids and gases by injecting the gas into the rotating, centrifugally stabilized liquid. Direct control of the strength of the centrifugal force permits the construction of very compact machines with high efficiency compared with conventional devices which rely on gravitational force for their operation. Since this force cannot be varied, such an apparatus must frequently be very large in order to process the necessary volume of gas. Thus, there are major expenses associated with the construction, transportation, and operation of these devices. High maintenance costs are also associated with the frequent removal of interior scale and deposits. With its drastically reduced size and simplicity of operation, vortex barbotage machinery offers significant reductions in costs, at all levels. In the condensed stable state (of the bistable states) of a gas-liquid mixture in a vortex chamber (figure 54a), a self-organized, rotating homogeneous layer of foam is formed near the periphery of the chamber (Goldshtik 1981). In this layer, the high centrifugal acceleration suppresses the entrainment of drops and increases the liquid-gas interface; this provides a very large reaction surface area per unit volume and enhances interfacial transport, further accentuated by turbulent mixing.

Principle of operation - Gas is injected from the walls into a swirling flow of liquid, forming a rotating bubbly or frothy layer. Extensive theoretical and experimental studies have determined the precise vortex chamber shape and other design parameters necessary to ensure a stable froth layer.

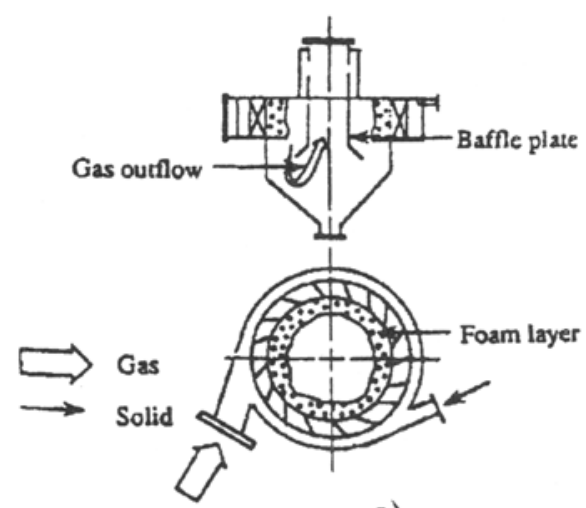

a)

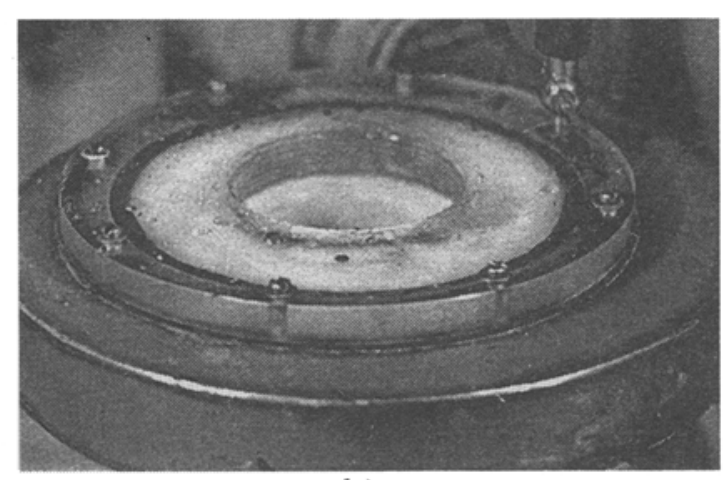

b)

Figure 54. Bubbling device and layer. 
Under these conditions, the stable froth layer maintains a liquid-gas force balance at the chamber walls as well as in the interior, ensuring uniformity in gas/liquid interaction as the gas passes through the liquid. A photograph of such a layer in a recent laboratory experiment is shown in figure $54 \mathrm{~b}$. The tangential injection of gas at the chamber walls through small nozzles creates bubble streams which then transfer their angular momentum to the liquid. Under normal operation, this generates centrifugal forces in the chamber which are 10 to 100 times greater than gravity. Such increased forces permit the use of much smaller diameter bubbles, which greatly increases the gas/liquid interfacial contact area. In addition, the very high speeds of the gas bubbles through the liquid and the strong secondary cross-flows in the liquid significantly enhance the liquid/gas interfacial interaction. Experiments have shown that a homogeneous froth layer can be maintained with correct design, without disruption by bubble coalescence or other nonuniformities. Due to these factors, a vortex barbotage apparatus can be built that can process the same volume of gas as a conventional device which is ten times its physical size and which consumes more energy. Here is a summary of the advantages of the vortex bubbling machine.

(i) Compared to conventional bubbling equipment, it is up to 10 times smaller and weighs as little as 1000 times less than comparable gas processing devices.

(ii) It has very high efficiency due to enhanced thermal and mass transfer processes.

(iii) Its small size and weight make transportation, and possible installation in existing facilities easy.

It can function with a wide range of gas and liquid flow ratios; thus the device can operate under strong inertial loading (e.g. on a moving vehicle), or in a micro-gravity environment (space applications); and works with the froth layer detached from the chamber walls; this reduces formation of scale and encrustation on walls, reducing maintenance costs.

\section{Possible applications include:}

(i) both particulate and vapour pollutant emission control at fossil fuel power generating plants. The worldwide market for such devices is in billions of dollars;

(ii) absorption and desorption of gases: e.g. carbon dioxide, nitrous oxide, oxygenation and carbonization of solutions, and many other chemical technological processes;

(iii) vaporization and condensation of liquids, such as in the thickening, heating and cooling of solutions, e.g. milk;

(iv) petroleum refining having a wide variety of applications;

(v) three-phase processes, in which the solid particle/liquid mixture is kept in a gas stream; this has important applications in the mining industry, e.g. flotation or segregation of fine gold from mine waste, and 


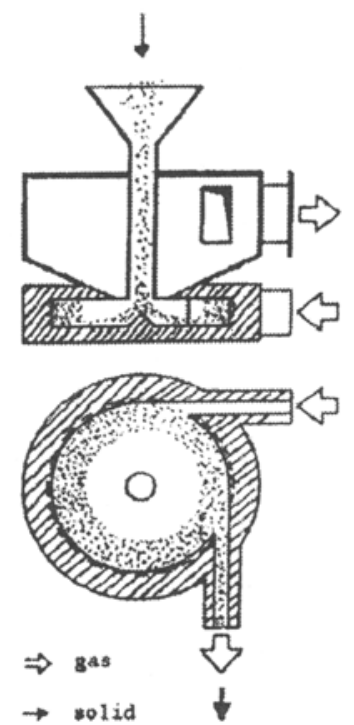

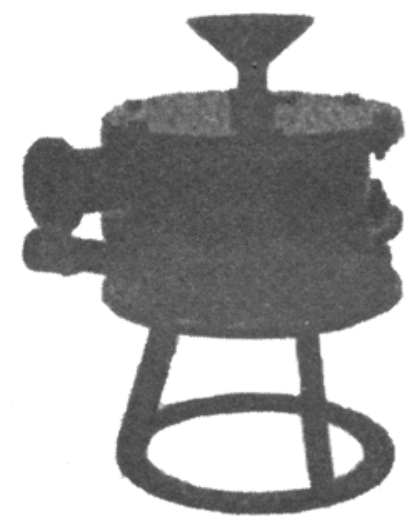

Figure 55. Vortex mill.

(vi) vortex nuclear reactor with a liquid uranium active zone. Because the critical temperature of liquid uranium is $\sim 20,000 \mathrm{~K}$, it is possible to heat the hydrogen working fluid up to temperatures of $6,000-7,000 \mathrm{~K}$, giving a specific impulse of $\sim 1,500 \mathrm{~s}$.

Vortex mill (VM): Theory and experiments (Goldshtik 1981, 1985) show that large particles can be ground efficiently while in the rarefied stable state (of the bistable states) of a solid-gas mixture in a rotating flow (figure 4). Adding acoustic resonators to the vortex chamber (figure 55) produces an intense sound field which considerably improves the grinding performance. We conjecture that this sound aids in grinding by preventing the healing of collision.cracks between successive impacts, thereby facilitating crack propagation. Operating parameters can be modified appropriately to allow extremely fine grinding, and thus VM has a very crucial use, e.g. in obtaining pure (99.9999\%) yttrium powder needed for making sintered film coatings for cathodes in the electronics industry. This is also an important ingredient in the manufacture of high-temperature superconductors. VM can also be used in cement production, and in the mixing of cement and fly ash in the manufacture of concrete slabs. In power plants, VM can permit on-site grinding of coal immediately prior to combustion, increasing combustion efficiency and eliminating off-site pulverizing to reduce transportation costs; in fact, it can be an integrated part of the combustor. VM can grind plastic as well as brittle material and thus can help grind used tyres for recycling, and polymers for use as additives for drag reduction in pipelines, submarines and boats etc. It can also be used as a very efficient liquid atomizer.

The vortex liquid piston engine project is sponsored by Advanced Research Project Agency (ARPA) under grant No. MDA972-93-1-0020. We are grateful to Dr Ed Carapezza for numerous technical discussions and Dr George Broze for reviewing the manuscript. 


\section{List of symbols}

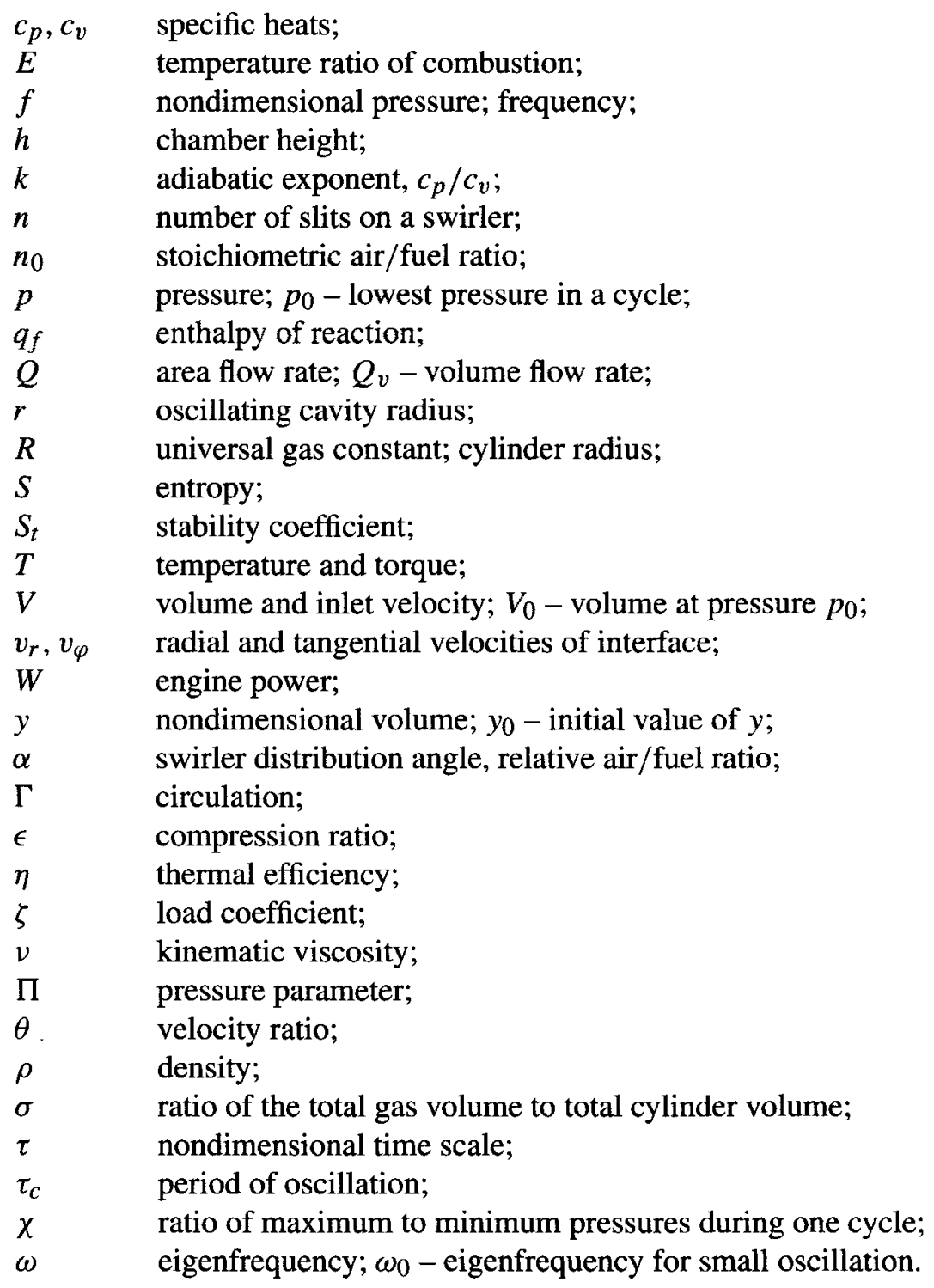

\section{References}

Batchelor G K 1967 An introduction to fluid dynamics (Cambridge: Cambridge University Press) Faires, Virgil M 1970 Thermodynamics (New York: MacMillan)

Goldshtik M A 1981a Vortex flows (Nauka: USSR Academy of Sciences)

Goldshtik M A 1981b Heat and mass transfer in a twisted gas-liquid layer. J. Appl. Mech. Tech. Phys. 22: 850-856

Goldshtik M A 1984 Transfer processes in granular layers (Nauka: USSR Academy of Sciences)

Goldshtik M A 1985 Variational model of a turbulent rotating flow. Fluid Dyn. 20: 353-362 
Goldshtik M A 1992 Engine employing rotating liquid as a piston. US Patent, No. 5,127,369

Greenspan H P 1969 The theory of rotating flows (Cambridge: University Press)

Gupta A K, Lilley D G, Syred N 1984 Swirl flows (Tunbridge Wells, UK: Abacus Press)

Hussain F 1986 Coherent structures and turbulence. J. Fluid Mech. 173: 303-356

Popular mechanics 1995 The incredible shrinking engine. January, p. 24

Shtern V, Hussain F 1995 Hysteresis in swirling jets. J. Fluid Mech. 309: 1-44

West C D 1983 Liquid piston stirling engines (New York: Van Nostrand Reinhold) 